\title{
H3K4 trimethylation dynamics impact diverse developmental and environmental responses in plants
}

\author{
Maryam Foroozani ${ }^{1}$, Matthew Vandal ${ }^{1}$, and Aaron Smith ${ }^{1}$ \\ ${ }^{1}$ Louisiana State University
}

April 28, 2020

\begin{abstract}
Plants execute developmental programs and respond to changing environmental conditions via adjustments in gene expression, which are modulated in part by chromatin structure dynamics. Histone modifications alter chromatin in precise ways on a global scale, having the potential to influence the expression of numerous genes. Trimethylation of lysine 4 on histone H3 (H3K4me3) is a prominent histone modification that is dogmatically associated with gene activity, but more recently has also been linked to gene repression. As in other eukaryotes, the distribution of H3K4me3 in plant genomes suggests it plays a central role in gene expression regulation, however the underlying mechanisms are not fully understood. Transcript levels of many genes related to flowering, root, and shoot development are affected by dynamic H3K4me3 levels, as are those for a number of stress-responsive and stress memory-related genes. This review examines the current understanding of how H3K4me3 functions in modulating plant responses to developmental and environmental cues.
\end{abstract}

\section{Introduction}

Eukaryotic DNA is complexed with histones into nucleosomes, which form the basic repeating unit of chromatin (Luger, Mäder, Richmond, Sargent, \& Richmond, 1997). Histone tails that protrude from nucleosome cores are subject to post-translational modifications, such as methylation, acetylation, ubiquitination, and phosphorylation, which alter chromatin structure in distinct ways. For example, histone methylation is an important modification involved in diverse processes including transcriptional regulation, heterochromatin formation, and DNA repair (Greer \& Shi, 2012; C. Liu, Lu, Cui, \& Cao, 2010). Methylation can occur on lysine or arginine residues with varying numbers of methyl groups, including mono-, di-, and trimethylation.

Trimethylated lysine 4 on histone H3 (H3K4me3) is a prominent histone mark whose biological importance is clear in light of phenotypic consequences of perturbing its deposition, such as poor chronological aging in yeast (Cruz et al., 2018), defective murine stem cell differentiation (Carlone \& Skalnik, 2001), and pleiotropic developmental defects in plants (Alvarez-Venegas \& Avramova, 2005; Guo, Yu, Law, \& Zhang, 2010b). Genome-level studies have revealed that H3K4me3 distribution is largely conserved among species and is enriched in genic regions, particularly around transcription start sites (TSSs; Barski et al., 2007; Bradley E Bernstein et al., 2002; X. Li et al., 2008; C. L. Liu et al., 2005; Ram et al., 2011; van Dijk et al., 2010; X. Zhang, Bernatavichute, Cokus, Pellegrini, \& Jacobsen, 2009). Also, H3K4me3 abundance is strongly correlated with transcript levels; hence, H3K4me3 has long been associated with gene activation (Françoise S Howe, Fischl, Murray, \& Mellor, 2017; Santos-Rosa et al., 2002). Despite these observations, the identification of clear causal relationships between H3K4me3 and gene expression are lacking. A recent study in yeast showed that H3K4me3 does not function in transcription initiation but modulates gene expression in other ways (Murray et al., 2019). On the other hand, Arabidopsis ATX1, a histone lysine methyltransferase (HKMT) that catalyzes H3K4 trimethylation, plays a dual role in gene expression, functioning in both transcription initiation and elongation (Ding, Avramova, \& Fromm, 2011b). 
Studies in diverse plant species and tissues have investigated genic levels of H3K4me3 in response to a variety of stresses (Foroozani et al., 2019; Jaskiewicz, Conrath, \& Peterhänsel, 2011; Kim et al., 2008; Pan et al., 2017; Sokol, Kwiatkowska, Jerzmanowski, \& Prymakowska-Bosak, 2007; Tsuji, Saika, Tsutsumi, Hirai, \& Nakazono, 2006; van Dijk et al., 2010; Zeng et al., 2019; Zheng et al., 2019). Generally these studies reported positive correlations between H3K4me3 abundance and stress-induced gene expression, further supporting an active role for H3K4me3. However, the genome-level studies showed large decreases in 5' localization of H3K4me3 in averaged genic profiles of stressed samples relative to controls (Foroozani et al., 2019; Zeng et al., 2019; Zong, Zhong, You, \& Xiong, 2013). Also, in two recent studies in rice, examination of specific subsets of stress-responsive genes revealed negative correlations between H3K4me3 abundance and gene upregulation in response to salt stress (Zheng et al., 2019) or phosphate (Pi) deficiency (Foroozani et al., 2019). These observations support a role for H3K4me3 in not only activation but also repression, consistent with findings in other systems (Clouaire et al., 2012; Françoise S Howe et al., 2017; Lenstra et al., 2011). Here we review the current understanding of H3K4me3 in plants, including the enzymes that alter its deposition in chromatin, how the mark influences gene expression and transcriptional memory, interactions between H3K4me3 and other modifications, and the specific developmental processes and stress responses that are modulated by H3K4me3 dynamics.

\section{H3K4 Methyltransferases}

Histone lysine methyltransferases (HKMTs) that methylate H3K4 contain an evolutionarily conserved SET ( $\mathrm{Su}(\operatorname{var}) 3-9$, Enhancer-of-zeste, and Trithorax) domain, which is required for catalytic activity (Shilatifard, 2012). In yeast (Saccharomyces cerevisiae), the Set1 HKMT is solely responsible for H3K4 methylation, whereasDrosophila melanogaster contains three Set1 orthologs: dSet1, Trithorax, and Trithorax-related (Roguev et al., 2001; Shilatifard, 2012). In plants, all HKMTs belong to the SET Domain Group (SDG). In Arabidopsis, the HKMTs are further categorized into seven classes based on function and domain structure (Ng et al., 2007; H. Zhou et al., 2020). The methyltransferases specific to H3K4 are members of class III, which is comprised of five Arabidopsis Trithorax-like (ATX) proteins, ATX1 through ATX5, and two ATX-related (ATXR) proteins, ATXR3 and ATXR7 (Avramova, 2009; Guo et al., 2010b; Tamada, Yun, chul Woo, \& Amasino, 2009). All class III proteins bear the SET and post-SET domains, but they contain varying combinations of other domains that allow for distinct functionality.

Perhaps the most studied of the ATX proteins is ATX1, which was found to methylate H3K4 at distinct genes in Arabidopsis (Alvarez-Venegas \& Avramova, 2005). The yeast Set1 methyltransferase must interact with structural proteins in a complex known as COMPASS (Complex Proteins Associated with Set1) to achieve H3K4 methylation (Schneider et al., 2005). The identification of an Arabidopsis COMPASS-like complex and its interaction with ATX1 confirms a similar scenario in plants (Ding, Ndamukong, et al., 2012a; D. Jiang, Kong, Gu, Li, \& He, 2011). ATX2 shows structural parallels with ATX1, but has divergent regulatory sequences that allow for differences in function. Whereas ATX1 catalyzes trimethylation at H3K4, ATX2 catalyzes dimethylation (Saleh et al., 2008). Also, ATX1 and ATX2 appear to regulate different subsets of genes (Saleh et al., 2008). ATXR3 (also named SDG2) is the predominant enzyme responsible for genomewide trimethylation at H3K4 (Guo et al., 2010b). The sdg2 mutant shows a pleiotropic phenotype, with stunted growth, impaired fertility, and differential expression of numerous genes (Guo, Yu, Law, \& Zhang, 2010a). In addition to trimethylation activity, SDG2 is capable of catalyzing dimethylation on H3K4, however, the significance of this function appears limited, as the sdg2mutant exhibited a minimal reduction in genomewide H3K4me2 (Guo et al., 2010b). ATX3, ATX4, and ATX5 are redundantly necessary for genome-wide di- and trimethylation of H3K4 at sites distinct from those targeted by ATXR3/SDG2 (L. Chen et al., 2017). Lastly, ATXR7 is able to methylate H3K4, but may also be involved in the dimethylation of H3K36 (Berr et al., 2009). In rice, five class III HKMTs have been described. Two of these, OsSDG723 (also named OsTrx1) and OsSDG701, are orthologs of ATX1 and ATXR3/SDG2, respectively, and contribute to H3K4 methylation levels (Choi et al., 2014; K. Liu, Yu, Dong, \& Shen, 2017).

\section{H3K4 Demethylases}

The dynamic nature of histone methylation became apparent after the identification of Lysine Specific Deme- 
thylase 1 (LSD1) in humans, which demethylates mono- and dimethyl H3K4 (Shi et al., 2004). Subsequently, proteins containing jumonji $\mathrm{C}(\mathrm{JmjC})$ domains $(\mathrm{JMJ})$ were identified as another family of histone demethylases. JMJ proteins can demethylate not only mono- and dimethylated, but also trimethylated substrates (Tsukada et al., 2006). Arabidopsis demethylases that target H3K4 include three homologs of LSD, FLOWERING LOCUS D (FLD), LSD1-LIKE1 (LDL1), and LSD1-LIKE2 (LDL2), and five JMJ proteins, JMJ14 through JMJ18 (P. Liu et al., 2019; Ueda \& Seki, 2020). An important aspect of H3K4 demethylase function appears to be its relationship with RNA-mediated DNA methylation (RdDM). H3K4me2 and H3K4me3 marks antagonize RdDM at many sites throughout the genome; therefore, demethylases (e.g. JMJ14, LDL1 and LDL2), act to maintain RdDM genomic patterns (Greenberg et al., 2013).

Arabidopsis H3K4 demethylases from both the LSD and JMJ families play important roles in regulating flowering. The three LSD demethylases have distinct and partially redundant roles in suppressing floral repressor genes (D. Jiang, Yang, He, \& Amasino, 2007). Similarly, JMJ14, JMJ15, and JMJ18 reduce H3K4me3 abundance at various flowering regulatory genes, thereby reducing their expression (Ning et al., 2015; H. Yang, Han, et al., 2012; H. Yang, Mo, et al., 2012). In addition to regulating the transition to flowering, Arabidopsis demethylases also play roles in RNA silencing (JMJ14; Searle, Pontes, Melnyk, Smith, \& Baulcombe, 2010), defense (JMJ14; D. Li et al., 2019), salinity stress (JMJ15; Shen et al., 2014), senescence (JMJ16; P. Liu et al., 2019), and dehydration stress (JMJ17; Huang et al., 2019). Rice demethylases that target H3K4 include OsJMJ701, OsJMJ703, and OsJMJ704 (X. Chen, Hu, \& Zhou, 2011; T. Song et al., 2018). OsJMJ701 and OsJMJ703 are involved in regulating flowering (Q. Chen et al., 2013; T. Song et al., 2018; Yokoo et al., 2014), whereas OsJMJ704 plays a role in modulating responses to a bacterial pathogen (Hou et al., 2015). Taken together, these studies indicate that demethylation of H3K4 at distinct loci is an important regulatory component of developmental and stress responses in plants.

\section{Functions of H3K4me3}

H3K4me3 is a prevalent mark conserved among species. Because it is enriched in genic regions and correlates with transcript levels, it is considered an active histone mark (Françoise S Howe et al., 2017; Santos-Rosa et al., 2002). However, H3K4me3 dynamics have been associated with not only activation, but also repression of subsets of genes (Clouaire et al., 2012; Foroozani et al., 2019; Françoise S Howe et al., 2017; Lenstra et al., 2011). Unlike its counterpart in yeast, Arabidopsis ATX1 has been shown to play a dual role in transcription (Ding et al., 2011b). Mutation of ATX1 led to substantial decreases in TATA Binding Protein (TBP) and RNA Polymerase II (Pol II) accumulation at the promoters of ATX1-target genes. This disruption of the transcription preinitiation complex (PIC) was associated with reduced target gene expression (Ding et al., 2011b). When ATX1 was mutated in only the SET catalytic domain, there was no observable decrease in the formation of the PIC (Ding, Ndamukong, et al., 2012b). This indicates that the deposition of H3K4me3 by ATX1 is not required for establishment of the PIC, but rather the formation of the ATX1/COMPASS-like complex is responsible for regulating transcription initiation. In addition to its recruitment to promoters, ATX1 is recruited to a promoter-proximal region where it catalyzes H3K4 trimethylation, which peaks at approximately 300-bp downstream of the TSS (Ding et al., 2011b). This recruitment is facilitated by the Ser5P (phosphorylated at serine 5 of the carboxyl terminal domain) form of Pol II, which is prevalent during early elongation. Continued elongation alters the phosphorylation state of Pol II to the Ser2P form, releasing ATX1 due to a lack of binding to this form. SET-domain mutants of ATX1 exhibit wild-type levels of Ser5P Pol II but have a considerable reduction in the level of Ser2P Pol II. This indicates that the rate of Pol II exit from initiation to elongation is reduced in the mutants (Ding et al., 2011b). These results indicate that ATX1, but not its H3K4 trimethylation activity, is required at promoters for PIC assembly and transcription initiation, whereas ATX1-generated H3K4me3 is essential for transcriptional elongation. Another aspect of gene expression subject to regulatory control is mRNA splicing. Successful mRNA splicing requires a 5'-cap, which is recognized and protected by a nuclear mRNA cap-binding complex (CBC; Calero et al., 2002). In Arabidopsis, the CBC was shown to interact with ATX and an H3K36 methyltransferase, indicating that trimethylation of both $\mathrm{H} 3 \mathrm{~K} 4$ and $\mathrm{H} 3 \mathrm{~K} 36$ is required for CBC-mediated 5'-cap preservation and mRNA splicing at ATX1 targets (Z. Li et al., 2016). Taken together these results demonstrate that H3K4me3 plays key roles in multiple aspects of gene regulation, including initiation, elongation, and mRNA splicing (Figure 
$1)$.

H3K4me3 has been implicated in the regulation of a number of developmental and environmental responses, and the associated mechanisms are subject to many functional considerations that are position- and/or context-dependent (Figure 2). In addition to the promoter versus promoter-proximal determinacy of ATX1 function described above, H3K4me3 at the transcription end site (TES) may play a role in antisense transcriptional control as observed for some mammalian genes (Cui et al., 2012). In this capacity, H3K4me3 may indirectly repress sense transcription by promoting antisense transcription. Further study is needed to determine the role of TES-localized H3K4me3 at plant genes. Another positional consideration of H3K4me3 function is the breadth of its distribution (i.e. peak width). A number of studies have suggested that relatively broad H3K4me3 peaks facilitate gene expression by reducing transcriptional noise or by promoting Pol II pausing (Park, Kim, Kwon, \& Lee, 2020). Also, genes marked by broad H3K4me3 domains tend to be responsive (i.e. with non-housekeeping functions) and exhibit cell type-specific functions. For example, Zhang et al. (2016), showed that broad H3K4me3 domains in chromatin isolated from human primary monocytes were biased at immune-responsive genes linked to the autoimmune disease, lupus. In Arabidopsis, H3K4me3 distribution at some dehydration stress-responsive genes was broader than at unresponsive genes (van Dijk et al., 2010). Therefore, broad H3K4me3 domains may recruit extensive, but paused, Pol II at stress-responsive genes, poising them for a robust transcriptional response upon induction.

In addition to positional considerations, H3K4me3 functions are also context-dependent. A study in yeast revealed that after cessation of transcription, H3K4me3 remained at promoter regions of several inducible genes and inhibited their reactivation (B. O. Zhou \& Zhou, 2011). Additionally, a more rapid transcriptional repression was observed at a number of genes previously repressed by carbon-source shifts, a phenomenon termed transcriptional repression memory (TREM; B. B. Lee et al., 2018). Induction of TREM is promoted by histone deacetylation via interaction between Rpd3L histone deacetylase (HDAC) and H3K4me3 (B. B. Lee et al., 2018). In Arabidopsis, H3K4me3 plays a role in the transcriptional memory of genes related to drought (Ding, Fromm, \& Avramova, 2012) and heat stress (Lämke, Brzezinka, Altmann, \& Bäurle, 2016). H3K4me3 levels significantly increased at response genes under re-exposure to stress treatments and displayed quicker and/or stronger transcriptional responses. In a related phenomenon, H3K4me3 accumulates at some defense-response genes during chemical priming. For example, exogenous application of the phytohormone salicylic acid (SA), or certain synthetic SA analogs, is sufficient to induce tolerance to a subsequent pathogen challenge (i.e. priming), and H3K4me3 appears to be involved in poising the primed defense-responsive genes for enhanced transcription (Kohler, Schwindling, \& Conrath, 2002; Mozgova et al., 2015a, 2015b).

Perhaps the foremost context-dependent consideration for the function of any chromatin mark is its interaction with other chromatin modifications. "Bivalent" nucleosomes or domains contain both activating and repressive marks, with the pairing of H3K4me3 and H3K27me3 in particular, is a prominent example (B. E. Bernstein et al., 2006). In mammalian H3K4me3-H3K27me3 bivalent domains, H3K4me3 poises genes for activation, whereas H3K27me3 represses transcription (B. E. Bernstein et al., 2006; Vastenhouw \& Schier, 2012). In Arabidopsis, H3K4me3-H3K27me3 bivalent domains were present at some genes required for gametophyte development (Berr et al., 2010), flowering time regulation (Qian et al., 2018), and at some dehydration stress-induced genes (N. Liu, Fromm, \& Avramova, 2014). Recently, a large number of genes in potato (Solanum tuberosum ) tubers up- or down-regulated by cold treatment were shown to contain the H3K4me3-H3K27me3 bivalent mark, suggesting it increases chromatin accessibility and facilitates recruitment of regulatory proteins necessary for either gene induction or repression (Zeng et al., 2019). More discussion on the interactions between H3K4me3 and other marks, as well as how H3K4me3 functions relate to specific developmental and environmental responses is presented in the sections below.

\section{H3K4me3 and Developmental Processes}

\subsection{Flowering Time and Flower Development}

Flowering is one of the most vital and regulated developmental processes in higher plants. Plants have evolved complex signaling pathways to ensure appropriate transition to the reproductive phase in accordance with 
developmental and environmental cues. In Arabidopsis, a number of components, including FLOWERING LOCUS C (FLC), the key floral repressor, its activator, FRIGIDA (FRI), and its downstream target, FLOWERING LOCUS T (FT), are involved in these pathways (I. Lee, Michaels, Masshardt, \& Amasino, 1994). Investigations of the molecular mechanisms of flowering have revealed the involvement of many H3K4 trimethyltransferases, largely through their role in modulating $F L C$ expression. Indeed, mutation of either of the ATX1 , ATX2 , ATXR7, or ATXR3 / SDG2 HKMTs directly or the COMPASS-like components, ASH2R or $R b B P 5$, resulted in reductions in H3K4me3 levels and transcription at $F L C$, along with an early flowering phenotype (Berr et al., 2009; D. Jiang et al., 2011; Michaels \& Amasino, 1999; Pien et al., 2008; Tamada et al., 2009; Yun, Tamada, Kang, \& Amasino, 2012). Notably, ATX1, ATXR7 and ASH2R were shown to directly bind the $F L C$ locus at the TSS. Since the expression level of $F L C$ is low in the native Colombia ecotype because of the absence of FRI, the function of H3K4 methyltransferases (ATX1, ATX2 ,ATXR7 and SDG2 ) in FLC activation were investigated in a Colombia background containing an active FRI allele. The activation of FLC by FRI was shown to be associated with H3K4me3 abundance and required ATX1 , ATX2 , ATXR7 and ATXR3activity (Pien, Fleury et al. 2008, Tamada, Yun et al. 2009, Yun, Tamada et al. 2012). Thus, COMPASS-like and H3K4 trimethylation are required for proper FLC expression and the vegetative to reproductive transition. Recently, characterization of the plant-specific protein, SHORT LIFE (SHL), revealed a flowering time regulatory mechanism that involves H3K4me3-H3K27me3 bivalent domains (Qian et al., 2018). SHL is capable of binding both H3K4me3 and H3K27me3, but in an independent and mutually exclusive manner. SHL negatively regulates the floral activator, SUPPRESSOR OF OVEREXPRESSION OF CONSTANS 1 (SOC1), likely by interacting with H3K4me3 and recruiting HISTONE DEACETYLASE 6 (HDA6) (Lopez-Gonzalez et al., 2014). Interestingly, the presence of H3K27me3 at the SOC1 locus is also necessary for SHL-mediated repression (Qian et al., 2018). Therefore SHL appears to be a central regulatory component in flowering time that mediates the switch between active and repressive states of SOC1, and likely other floral integrator genes. Given that overexpression or antisense-knockdown of $S H L$ resulted in a pleiotropic phenotype, including perturbations in growth, senescence, and flower and seed formation (Mussig, Kauschmann, Clouse, \& Altmann, 2000), SHL is likely to modulate a number of distinct developmental pathways.

Further evidence for a role of H3K4me3 in flowering time regulation has been determined through investigations of demethylases. JMJ15 and JMJ18 contribute to floral activation by decreasing H3K4me3 and expression of $F L C$, whereas JMJ14 is implicated in floral repression by decreasing H3K4me3 and expression of FT (Jeong et al., 2009; Ning et al., 2015; H. Yang, Han, et al., 2012; H. Yang, Mo, et al., 2012; W. Yang, Jiang, Jiang, \& He, 2010). However, two additional studies showed that mutation of JMJ14 caused changes in the expression levels of multiple floral regulator-encoding genes, including $F T$,SOC1, LFY, and AP1 , without corresponding changes in H3K4me3 levels (Falong Lu, Cui, Zhang, Liu, \& Cao, 2010; Ning et al., 2015). Additional studies are needed to resolve the mechanistic details of JMJ14 function at floral regulator genes. JMJ14 appears to influence flowering, in part, via physical interaction with two NAC (NAM, ATAF1, and CUC1/CUC2) transcriptional repressors, NAC050 and NAC052 (Ning et al., 2015). Mutation of JMJ14 or RNAi knock-down of NAC050 and NAC052 led to an early flowering phenotype, as well as up-regulation and enhanced H3K4 trimethylation at a number of target genes. Therefore, JMJ14 and NAC050/NAC052 interact and co-regulate a number of genes via H3K4 demethylation and transcriptional repression, including genes that influence flowering time control.

H3K4me3 is also required for proper flowering time in rice. The H3K4me3/H3K27me3 ratio and expression levels for a number of genes were correlated during transition from the shoot apical meristem (SAM) to the inflorescence meristem (IM; X. Liu et al., 2015). Specifically, the H3K4me3/H3K27me3 ratio was relatively high and low at up- and down-regulated genes, respectively. Also, mutations in OsSDG711(H3K27 methyltransferase) or OsJMJ703 (H3K4 demethylase) were found to reduce induction in the levels of H3K27me3 at many genes in the IM. H3K4me3 and H3K27me3 levels were increased and decreased, respectively, in both the osjmj703 and ossdg711 single mutants at the CKX2/Gn1a locus, a gene expressed in IM related to panicle size. The ossdg711 osjmj703 double mutant showed strong induction of H3K4me3 and reduction of H3K27me3, suggesting that OsSDG711 and OsJMJ703 function to control the H3K4me3/H3K27me3 ratio 
(Liu, Zhou et al. 2015). A recent study utilizing cell-type specific analysis with stem cells and non-stem cells found that within the SAM, H3K4me3 and H3K27me3 levels changed during floral transition, with an increase in H3K4me3 corresponding to an increase in gene expression (You et al., 2017). However, the results of this study suggest that the level of H3K27me3 does not predict levels of gene expression in the SAM as a result of flowering. OsTrx1 and OsSDG701, rice orthologs of ATX1 and ATXR3/SDG2, respectively, also play roles in regulating flowering time. OsTrx1 binds to Early Heading Date 3 (Ehd3), which induces flowering through downregulation of Heading Date 7(Ghd7 ; Choi et al., 2014). In addition, OsSDG723/ OsTrx1/ OsSET33 Interaction Protein 1 (SIP1) recruits OsTrx1 to Ehd1 where it impacts H3K4me3 levels and expression (P. Jiang et al., 2018). Ehd1, in turn, regulates the florigen-encoding genes, Heading Date 3a (Hd3a) and Rice Flowering Locus T1 (RFT1). In ossdg701 mutants, the expression of all three genes was downregulated, and H3K4me3 levels were reduced at Hd3a and RFT1, but not Ehd1 (K. Liu et al., 2017). This indicates that OsSDG701 is necessary for the transition to flowering, apparently through direct and indirect interactions with floral regulators.

Three studies have investigated the roles of H3K4 demethylases (OsSe14 and OsJMJ703) in rice flowering and development (Q. Chen et al., 2013; T. Song et al., 2018; Yokoo et al., 2014). Mutation of osse14caused early flowering, increased levels of H3K4me3 at RFT1, and induced RFT1 expression (Yokoo, Saito et al. 2014). A more recent study showed that mutation of OsJMJ703 caused early flowering, and overexpression of OsJMJ703 changed the structure of the panicle (T. Song et al., 2018). The levels of H3K4me3 were measured in both mutant and overexpression lines, and the developmental defects were associated with higher and lower levels of H3K4me3, respectively.

In addition to a role in regulating flowering time, H3K4me3 dynamics have been linked to flower development. Mutation of Arabidopsis ATXR3 /SDG2 resulted in floral defects including severe male and female reproductive organ abnormalities, as well as inability to maintain indeterminate growth of the IM, resulting in a terminal flower (Guo et al., 2010b). Similarly, mutation of Arabidopsis ATX1 led to defects in floral organ development and identity (Alvarez-Venegas et al., 2003). In situ hybridization assays showed a correlation between ATX1 expression and floral organ initiation. Additionally, the expression of three homeotic genes, APETALA (AP1,AP2 ), and AGAMOUS ( $A G$ ) were reduced in atx1 mutants. Dynamic change of H3K4me3 was found to be a strong indicator for changes in gene expression over flower development, moreso than H3K27me3, although several genes were found to be marked differentially by these two modifications throughout development (Engelhorn et al., 2017). During flower initiation, changes in H3K4me3 occurred sooner and exhibited a stronger correlation with expression relative to H3K27me3. It is likely that bivalent domains containing both H3K4me3 and H3K27me3 marks modulate expression of many genes in accordance with developmental cues.

\subsection{Root and Shoot Development}

Trimethylation of H3K4 is required for proper root and shoot development, which is exemplified by the pleiotropic phenotypes of HKMT mutants. In addition to many floral defects, Arabidopsis sdg2 andatx1 mutants exhibit small shoot and root organs (Alvarez-Venegas \& Avramova, 2005; Guo et al., 2010b; NapsucialyMendivil, Alvarez-Venegas, Shishkova, \& Dubrovsky, 2014; Yao, Feng, Yu, Dong, \& Shen, 2013). Detailed characterization showed that loss of SDG2 function and reduction of H3K4me3 levels in root cells led to a smaller root apical meristem (RAM), disorganized root stem cell niche, and as a consequence, halted meristem activity in both primary and lateral roots (Yao et al., 2013). In addition to loss of cell identity and stem cell function, there was no observable auxin gradient in the root quiescent center within sdg2 mutants. More similarities between sdg2and atx1 mutants include delayed primary root growth, decreased RAM size and activity, and attenuated lateral root emergence. Interestingly, the transition from cell proliferation to cell elongation in the RAM was impaired in mutants of ATX1, but not SDG2 , revealing non-redundant requirements of ATX1 for root development (Napsucialy-Mendivil et al., 2014).

Evidence suggests H3K4me3 is involved in shoot development by regulating the expression of the repressive shoot developmental genes, MIR156A and MIR156C (Xu, Leichty, Hu, \& Poethig, 2018). Transition from juvenile to adult vegetative phase is controlled by induction of SQUAMOSA PROMOTER BINDING 
PROTEIN-LIKE (SPL) expression, which is negatively regulated by miR156A and miR156C (Poethig, 2010; Yu et al., 2013). In wild-type plants at theMIR156A and MIR156C genes, H3K4me3 levels decreased gradually during shoot development whereas H3K27me3 levels increased (Xu et al., 2018). Mutation in ACTIN-RELATED PROTEIN 6(ARP6), which facilitates deposition of the H2A.Z variant, reduced the level of H3K4me3 at both genes, whereas nucleosome occupancy and H3K27me3 increased at only MIR156A (Xu et al., 2018). Additionally, expression levels of MIR156A and MIR156Cwere reduced in arp6 mutants. These results indicate that genes regulated by H2A.Z-assisted deposition of H3K4me3 are required for shoot development in Arabidopsis (Xu et al., 2018).

H3K4me3 dynamics have also been implicated in tissue identity and plant regeneration. For example, Arabidopsis ATX4 was shown to regulate shoot identity genes required for successful transition from callus to shoot tissue (K. Lee, Park, Choi, \& Seo, 2019). In rice, H3K4me3 was found to be important for stem elongation and growth. Mutants of OsJMJ703exhibited semi-dwarf phenotypes due to slower cell division and cytokinin deficiency, apparently as a result of increased H3K4me3 at the promoter of CYTOKININ OXIDASE (CKX) and induction of CKX expression (Q. Chen et al., 2013).

\subsection{Sporophyte and Gametophyte Development}

Berr et al. (2010) reported on the involvement of ATXR3/SDG2 in sporophyte and gametophyte development in Arabidopsis. Results indicated that leaf sizes were reduced during the vegetative phase in thesdg2 mutant because of impaired cell division and cell expansion. Sterility of the $s d g 2$ mutants was caused by defective anther and pollen development along with short stamens and misregulated microgametogenesis. In addition, megagametogenesis was halted in mostsdg2 mutant ovules, leading to degenerate megaspore nuclei and defective embryo sacs. The expression levels ofSPOROCYTELESS/NOZZLE (SPL/NZZ) and MALE STERILITY1(MS1), two essential genes for male and/or female gametophyte development, were drastically reduced in sdg2 mutants. In wild-type plants, $S P L / N Z Z$ and $M S 1$ contain H3K4me3-H3K27me3 bivalent nucleosomes. In $s d g 2$ mutants, the levels of H3K4me3, but not H3K27me3, were decreased. Therefore, SDG2-mediated H3K4me3 deposition regulates expression of two essential developmental genes, controlling molecular mechanisms for gametophyte development. More recently, SDG2 was shown to play a role in postmeiotic gametogenesis by promoting chromatin decondensation, mitotic cell division, and activation of the ATLANTYS1 retrotransposon (Pinon, Yao, Dong, \& Shen, 2017). At the tricellular stage, sdg2 pollen grains contained only two sperm nuclei per pollen. Also, chromatin was more condensed in the sdg2 mutant as compared to wild type. This elevated chromatin compaction in sdg2 resulted in defects in ATLANTYS1 activity, pollen germination, and pollen tube elongation, which were accompanied by a reduction of H3K4me3 and reorganization of $\mathrm{H} 3 \mathrm{~K} 9 \mathrm{me} 2$.

\subsection{Circadian Clock}

Rhythmic transition from activation to repression of oscillator genes within the circadian clock is influenced by H3K4me3 dynamics. Both the expression of Arabidopsis oscillator genes responsible for circadian clockwork and the levels of H3K4me3 at those genes are dependent on SDG2 (Malapeira, Khaitova, \& Mas, 2012). A decrease in H3K4me3 levels by nicotinamide treatment showed an induction in the binding of a clock transcriptional repressor, PSEUDO-RESPONSE REGULATOR 5 (PRR5), to its target genes, causing repression. In wild type, the enrichment of H3K4me3 and H3K56ac was higher at evening-expressed oscillator genes at Zeitgeber Time 15 (ZT15), whereas a reduction in the level of both histone modifications was observed at morning-expressed oscillatory genes at ZT15. The opposite was true for ZT3, with H3K4me3 and H3K56ac increasing at morning-expressed oscillator genes, while decreasing at the evening genes. A time course analysis showed that H3K4me3 had a delayed accumulation when compared to H3K56ac, suggesting a difference in accrual could be specific to time-related activating functions. In addition, H3K4me2 levels were high at the time H3K56ac was low and vice versa, whereas the coexistence of H3K4me3 and H3K4me2 was also detected. This suggests a sequential accumulation of first H3K56ac, then H3K4me3, and finally H3K4me2, which is temporally associated with the expression of oscillator genes through the circadian cycle (Malapeira et al., 2012). 


\subsection{Leaf Senescence}

The dynamics of several histone marks, including H3K4me3, H3K27me3, and H3K9ac, have been implicated in the regulation of gene expression during leaf senescence. Genome-wide investigation showed in older leaves of Arabidopsis a positive correlation between H3K4me3 and transcript levels, as well as a negative correlation between H3K27me3 and transcript levels (Brusslan et al., 2012). Only a limited portion (5\%) of differentially expressed genes showed changes in both H3K4me3 and H3K27me3, suggesting the importance of H3K4me3H3K27me3 bivalent domains in the developmental transition of senescing leaves may be minimal (Brusslan et al., 2012). In a subsequent study, a stronger positive correlation between H3K4me3 levels and gene expression was shown during leaf senescence (Brusslan et al., 2015). Around 56\% of increased H3K4me3 genes and $63 \%$ of decreased H3K4me3 genes were up- and down-regulated, respectively, during leaf senescence, whereas a moderate positive correlation was observed between H3K9ac and gene expression. Recently, Liu et al. (2019) investigated the mechanism of how age-dependent induction of senescence-associated genes (SAGs) are associated with high levels of H3K4me3. Rice osjmj16 mutants displayed a premature senescence phenotype, as well as an increase in both H3K4me3 abundance and expression levels of several SAGs, such as WRKY53 and SAG201 . In addition, OsJMJ16 expression decreased in the later stages of leaf development. Thus, OsJMJ16 appears to negatively controls leaf senescence, in part, by repressing the expression of WRKY53 and SAG201.

\section{H3K4me3 and Stress Responses}

\subsection{Drought Stress}

Drought is one of the most prominent and growth-limiting abiotic stressors encountered by crop plants. In response to dehydration, plants initiate signaling pathways aimed at enhancing tolerance to drought via molecular, biochemical, and physiological responses. More than a decade ago, H3K4me3 was first implicated in regulating plant responses to drought. By examining chromatin marks specifically at four drought-inducible genes in Arabidopsis (RD29A , RD29B ,RD20 , and RAP2.4 ) Kim et al. (2008) showed that H3K4me3 abundance, along with transcript levels, increased at all four genes in response to drought stress (Kim et al., 2008). Subsequent genome-wide studies in Arabidopsis (van Dijk et al., 2010) and rice (Zong, Zhong et al. 2013) showed a similar trend in which many genes up- or down-regulated by dehydration exhibited increased and decreased H3K4me3 abundance, respectively.

Examination of transgenic lines exhibiting altered expression of methylases and demethylases suggest direct involvement of H3K4me3 dynamics in controlling responses to drought stress. Mutation of ATX1 in Arabidopsis (Ding, Avramova, \& Fromm, 2011a) or overexpression of OsJMJ703 in rice (Song, Zhang et al. 2018) each led to transgenic lines that exhibited increased sensitivity to drought stress. Consistently, RNAi knockdown of OsJMJ703 conferred enhanced drought tolerance (Song, Zhang et al. 2018). On the other hand, in wild-type plants, OsJMJ703 expression was reduced by drought stress, whereas ATX1 expression was unaffected (Ding et al., 2009). This suggests different roles for H3K4me3 methyltransferases and demethylases in drought stress responses, such that the catalytic activity of methyltransferases (e.g. ATX1) is responsive to stress, but demethylases (e.g. OsJMJ703) respond to stress through increases in their gene expression.

Detailed characterization of Arabidopsis atx1 mutants revealed that the increased sensitivity of drought conferred by loss of ATX1 was in part due to reduced biosynthesis of abscisic acid (ABA), an important phytohormone that modulates osmotic stress pathways in plants (Ding et al., 2011a). Specifically, dehydration stress increased transcription and H3K4me3 abundance at the NCED3 ABA biosynthetic gene, which required recruitment of ATX1 to theNCED 3 locus. ATX1, in turn, increased the amount of Pol II bound to NCED3 . Therefore, ATX1 influences drought-stress pathways by upregulating NCED 3 transcription and ABA production (Ding et al., 2011a).

Characterization of the NCED3 locus also highlights two distinct functions of ATX1 in Arabidopsis (Ding et al., 2011a). In the promoter, ATX1 aids in transcription initiation by recruiting TBP and Pol II. Upon transition to a transcriptional elongation state, phosphorylation occurs at Ser5 of Pol II's carboxy-terminal 
domain (CTD). ATX1 is recruited to Ser5P-containing regions, which peak at around +300 bp downstream of the TSS, at which it catalyzes H3K4 trimethylation. Additional transcriptional elongation leads to the predominantly Ser2P form of CTD, resulting in loss of ATX1. These data indicate that ATX1 plays distinct roles in Pol II recruitment and H3K4 trimethylation, both of which appear to be involved in the regulation of NCED3 during drought stress. The observation that the level of H3K4me3 was established only after Pol II accumulation at two drought-responsive genes (RD29Aand RAP2.4) further supports a model in which Ser5P-dependent H3K4 trimethylation occurs subsequently to Pol II recruitment in the promoter (Kim et al., 2008).

The H3K4me3 distribution profile at some dehydration stress-responsive genes has been shown to be broader than unresponsive genes, although these profiles are present during both control and dehydration stress conditions (van Dijk et al., 2010). At human tumor-suppressor genes, broad H3K4me3 peaks were linked to increased transcription elongation (K. Chen et al., 2015). Although the relevance of H3K4me3 breadth is not completely clear, the broad H3K4me3 peaks at drought-responsive genes (and possibly other stressresponsive genes) may reflect extensive gene-body H3K4 trimethylation, and perhaps extensive Ser5P Pol II, to ensure a transcriptionally poised state in preparation for stress induction.

A particularly striking phenomenon that has been revealed through investigations of H3K4me3 in drought stress responses is its contribution to transcriptional memory. Based on their expression pattern during reoccurring drought stress, four dehydration-inducible marker genes, RD29A, RD29B, RAB18, and COR15A , were designated 'trainable' or 'non-trainable' (Ding, Fromm, et al., 2012). Transcripts of trainable genes ( $R D 29 B, R A B 18$ ) were greater during recurring stress relative to their levels upon the initial stress; however, transcript levels of non-trainable genes (RD29A , COR15A) were induced at the same level during each stress. Despite this difference, expression of both trainable and non-trainable genes returned to initial levels during the recovery states. For non-trainable genes, the degree of H3K4me3 enrichment was similar after each stress treatment, but in trainable genes, H3K4me3 was significantly increased in repetitive stress treatments. In the recovery states, the levels of H3K4me3 and Ser5P Pol II remained high in trainable genes even with the low level of transcription, showing Ser5P Pol II was stalled. These data suggest that the presence of H3K4me3 may be necessary for elongation but does not guarantee the transition from initiation to elongation. H3K4me3 behaves as an adjustable chromatin mark which is correlated with transcription levels in non-trainable genes, but acts as an epigenetic memory mark that is not removed during recovery states in trainable genes (Ding, Fromm, et al., 2012).

\subsection{Temperature Stress}

Another example of H3K4me3-mediated transcriptional memory occurs during heat stress. Plants exposed to moderate heat stress acquire thermotolerance, acclimating them to a subsequent high temperature stress that is lethal to non-acclimated plants (Mittler, Finka, \& Goloubinoff, 2012). The acquired thermotolerance is maintained for several days after a return to non-stress temperatures, a phenomenon termed heat-stress memory. Transcriptomic analysis identified a number of putative heat-stress memory genes based on their persistent elevated transcription for several days after the heat stress (Stief et al., 2014). These genes contrast with heat stress-induced genes whose expression declines relatively quickly (i.e. heat-stress non-memory genes). Investigating the levels of $\mathrm{H} 3 \mathrm{~K} 4 \mathrm{me} 2 / 3$ at the representative heat-inducible memory and non-memory genes, HEAT SHOCK PROTEIN (HSP ) 22.0 and 70 , respectively, showed that these marks play a role in heat-stress memory (Lämke et al., 2016). Relatively soon after an acclimatizing heat stress, the non-memory gene $\left(H S P^{70}\right)$ exhibited a moderate increase in H3K4me3 that declined over the next several days. In contrast, the memory gene (HSP22.0 ) showed a dramatic increase in H3K4me3 that remained for several days. Also, H3K9 acetylation (H3K9ac) was highly enriched early at both loci, and declined rapidly at $H S P^{70}$ , but declined relatively slowly at HSP22.0 , maintaining appreciable levels for several days. Further, HSP70 exhibited no changes in H3K4me2, whereas H3K4me2 levels increased at HSP22.0 , but only at the later time points of the experiment. These patterns of H3K4me2/3 and H3K9ac dynamics at the HSP22.0 memory gene are reminiscent of the mechanism that establishes transcriptional memory at some yeast genes, such as INO1 , which encodes inositol-1-phosphate synthase. Inositol starvation induces transcription of INO1, which is 
accompanied by increases in H3/H4 acetylation and H3K4 di- and trimethylation (D'Urso et al., 2016). As memory is induced, levels of acetylated $\mathrm{H} 3 / \mathrm{H} 4$ and $\mathrm{H} 3 \mathrm{~K} 4 \mathrm{me} 3$ decline, whereas $\mathrm{H} 3 \mathrm{~K} 4 \mathrm{me} 2$ levels persist. The shift in H3K4 methylation results from a remodeled version of the COMPASS complex that catalyzes H3K4 mono- and demethylation, but not trimethylation. Interestingly, the sustained accumulation of $\mathrm{H} 3 \mathrm{~K} 4 \mathrm{me} 2 / 3$ at Arabidopsis HSP22.0 required HSFA2, a transcription factor necessary for heat-stress memory, but not heat-stress responses in naïve plants. These findings support a model for plant transcriptional memory in which stress-specific transcriptional activators such as HSFA2 bind memory genes and help modulate H3/H4 acetylation and H3K4me di- and trimethylation dynamics similar to those at yeast memory genes.

A recent study investigated the impact of cold stress on the chromatin environment of potato by comparing maps of DNase I hypersensitive sites (DHSs) for tubers stored at room temperature or cold $\left(4^{\circ} \mathrm{C}\right)$ conditions (Zeng et al., 2019). DHSs are indicative of relatively accessible genomic regions that contain active cis-regulatory DNA elements. In response to cold stress, a large number of DHSs were induced at genic regions associated with temperature-responsive genes. Interestingly, the H3K4me3-H3K27me3 bivalent mark was present in the gene bodies of many of these genes, and those that were up-regulated were linked to stress responses, whereas those down-regulated were linked to developmental processes. This suggests that the H3K4me3-H3K27me3 bivalent mark facilitates enhanced accessibility during stress and allows for the recruitment of activators or repressors in a gene-function dependent manner.

\subsection{Salt Stress}

Investigations of a number of stress-responsive genes in Arabidopsis have shown that several chromatin marks impact the responsiveness of ABA- and salt-induced genes. Both $\mathrm{ABA}$ and salt stress led to enrichment of H3K4me3 and H3K9K14 acetylation, but decreased H3K9me2 at a subset of ABA- and salt stress-responsive loci (L.-T. Chen, Luo, Wang, \& Wu, 2010). Mutation of HISTONE DEACETYLASE 6(HDA6 ) led to increased $\mathrm{H} 3$ acetylation at ABA/salt-inducible genes compared to wild type under control conditions. In response to $\mathrm{ABA}$, wild type, but not HDA6 mutants, exhibited enriched $\mathrm{H} 3$ acetylation at $\mathrm{ABA}$-induced loci. Interestingly, mutation of HDA6also abolished the enrichment of H3K4me3 exhibited at ABA-induced genes in wild type. This suggests that $\mathrm{H} 3$ deacetylation by HDA6 is necessary for H3K4me3 enrichment at ABA-induced loci. (L.-T. Chen et al., 2010). Similarly, in soybean, three salt-stress inducible genes encoding transcription factors showed induction in $\mathrm{H} 3 \mathrm{~K} 4 \mathrm{me} 3$ and reduction in both H3K9me2 and DNA methylation in response to salinity stress (Y. Song et al., 2012).

A recent study by Zheng et al. (2019) showed that salt stress alters the distribution of H3K4me3 in a tissue specific manner. In response to salt, rice seedlings exhibited H3K4me3 decreases and increases in exons and introns, respectively, whereas only exonic decreases in H3K4me3 were observed in roots (Zheng et al., 2019). Notably, a chromatin state defined by containing H3K3me3-H3K27me3 bivalent marks correlated with both up- and down-regulated genes in seedlings, but only down-regulated genes in roots. In potato tubers the H3K3me3-H3K27me3 bivalent mark was enriched at genes both up- and down-regulated by cold stress (Zeng et al., 2019). Together these observations may reflect a stronger and more biased cessation of developmental processes in salt-stressed roots relative to whole seedlings.

H3K4me3 association with salt stress was further demonstrated by studying H3K4 demethylases in Arabidopsis (Shen et al., 2014). During salt stress, JMJ15 expression increased, whereas JMJ14 andJMJ18 expression decreased. Analysis of $j m j 15$ mutants showed a reduction in salt stress tolerance, whereas JMJ15 -overexpression led to increased tolerance. Examination of global H3K4me1/2/3 data showed that a majority of genes down-regulated by JMJ15 -overexpression were marked with H3K4 di- and trimethylation, but up-regulated genes did not show the same H3K4me2/3 bias. This suggests that JMJ15 represses gene expression at salt-responsive genes via H3K4 demethylation.

\subsection{Submergence Stress}

In rice, submergence stress induced changes in the levels of H3K4 methylation at the submergence-inducible genes, ADH1 andPDC1 (Tsuji et al., 2006). Specifically, during submergence, the levels of H3K4me3 and H3K4me2 increased and decreased, respectively, at both 5' and 3' genic regions. This finding suggests 
submergence stress results in conversion of H3K4me2 to H3K4me3, which may recruit Pol II and lead to increased transcript levels. In addition, during submergence stress, the level of $\mathrm{H} 3$ acetylation showed a gradual increase that occurred after the levels of Pol II and transcripts increased. This suggests an important temporal interaction between $\mathrm{H} 3$ methylation and acetylation dynamics that modulates the response to submergence stress.

\subsection{Nutrient Stress}

Studies investigating responses to phosphate $(\mathrm{Pi})$ starvation and high nitrogen have indicated a role for H3K4me3 in plant nutrient homeostasis. A recent report on chromatin dynamics in Pi-starved rice shoots showed that over $40 \%$ of protein-coding genes exhibited a low-Pi change in chromatin states (CSs) defined by differing levels of H3K4me3, H2A.Z, and nucleosome occupancy at their TSS (Foroozani et al., 2019). Genes marked by H3K4me3 and H2A.Z that exhibited decreases in both marks in response to Pi starvation were enriched among low-Pi induced genes. This suggests that combined loss of H3K4me3 and H2A.Z accompanies de-repression of some Pi-starvation inducible genes. On the other hand, genes marked by H3K4me3 and H2A.Z that exhibited a decrease in only H3K4me3 were enriched among both up- and down-regulated genes. This suggests a possible dual role of H3K4me3 in modulating expression of this subset of Pi-responsive genes, of which a majority were linked to cell wall and lipid remodeling pathways. A third subset of genes, which included many translation-related loci including ribosomal protein genes, suggested a pair of distinct CS transitions at the +1 and -1 nucleosomes. Under control conditions, these genes contained low H2A.Z and high H3K4me3 at the -1 nucleosome, but high levels of both marks at the +1 nucleosome. Pi starvation led to specific losses of H3K4me3 at the -1 nucleosome and H2A.Z from the +1 nucleosome. Interestingly, mutation of the yeast COMPASS component Spp1 results in substantial decreases in global H3K4me3, but the genes that retain disproportionately high H3K4me3 levels are enriched in ribosomal protein genes and other translation-related genes (F. S. Howe et al., 2014). This suggests that in rice, as in yeast, multiple COMPASS-like complexes with different subunit compositions target distinct subsets of genes.

A role for H3K4me3 in Pi-starvation responses was also demonstrated in Arabidopsis with the characterization of the ALFIN-LIKE 6(AL6) gene, which encodes an H3K4me3-binding protein (W. Y. Lee, Lee, Chung, \& Kwon, 2009). Mutation of $A L 6$ resulted in a pleiotropic phenotype including inhibition of low-Pi induced root hair elongation (Chandrika, Sundaravelpandian, Yu, \& Schmidt, 2013). Predicted targets of AL6 include ENHANCER OF TRY and CPC1(ETC1), which encodes a transcriptional activator of Piresponsive root hair elongation genes (Chandrika et al., 2013). Thus, AL6 appears to regulate ETC1 through its interaction with H3K4me3 by promoting transcription elongation and/or mRNA maturation.

To maintain optimal nitrogen levels, plants repress root nitrate uptake in response to high nitrogen conditions. The characterization of thehigh nitrogen-insensitive 9 ( hni9) mutant revealed that HNI9 encodes INTERACT WITH SPT6 (IWS1), a conserved component of the Pol II complex (Widiez et al., 2011). In response to high nitrogen supply, the transcription of the NRT2.1 nitrate transporter gene was repressed, which was associated with HNI9/IWS1-dependent H3K27 trimethylation across the NRT2.1 locus from promoter to 3' region. This increase in H3K27me3 coincided with a decrease in H3K4 trimethylation across the gene body, but not at the promoter, where H3K4me3 levels were unchanged. Recently, HNI9/IWS1 was also shown to influence nitrogen homeostasis by modulating the detoxification of reactive oxygen species (ROS) induced by high nitrogen (Bellegarde et al., 2019). Mutation ofHNI9 / IWS1 blocked the high-nitrogen induction of a number of ROS-detoxification genes. Interestingly, H3K4me3 levels were lower at these genes in the hnig mutant relative to wild type, whereas levels of H3K27me3, H3K36me3, and H3K9ac were unchanged. This suggests that HNI9/IWS1 induces detoxification genes in order to quench high nitrogen-induced ROS by facilitating H3K4me3 accumulation. HNI9/ISW1 is known to interact with brassinosteroid-related factors that facilitate demethylation of H3K27me3 and trimethylation of H3K36, thereby acting as a transcriptional switch from a repressed to active state (Crevillen et al., 2014; Y. Li et al., 2015; F. Lu, Cui, Zhang, Jenuwein, \& Cao, 2011). In a similar mechanism, HNI9/ISW1 may interact with factors to increase the H3K4me3/H3K27me3 ratio at ROS detoxification genes in response to high nitrogen.

\subsection{Biotic Stress}


In addition to a role for $\mathrm{H} 3 \mathrm{~K} 4 \mathrm{me} 3$ in a number of abiotic stress responses, this histone mark has also been implicated in responses to biotic stress, particularly during plant-pathogen interactions. During pathogen exposure, H3K4me3 levels have been shown to increase at defense-related genes. For example, Botrytis cinerea infection in both Arabidopsis and tomato plants resulted in increased levels of H3K4me3 at upregulated genes (Alonso, Ramos-Cruz, \& Becker, 2019; Roberts \& Sanchez, 2019). In contrast, the level of H3K4me3 was not significantly altered at down-regulated genes.

Analyses of H3K4 methyltransferase and demethylases have further demonstrated a role for H3K4me3 in plant defense responses. $W R K Y^{\gamma} 0$, a transcription factor involved in plant defense responses, was shown to be a direct target of ATX1, while also being positively regulated by ATX1-generated H3K4me3 (AlvarezVenegas, Abdallat, Guo, Alfano, \& Avramova, 2007). Mutation of another Arabidopsis HKMT, ATXR7, led to greater sensitivity to the pathogen, Hyaloperonospora arabidopsidis (Xia et al., 2013). A suppressor screen for the suppressor of npr1-1, constitutive 1 (snc1) mutant, which exhibits constitutive activation of plant defense responses, identified modifier of snc1,9 (mos9). MOS9 was shown to directly interact with ATXR7, and mos 9 mutants exhibited reductions in H3K4me3 and transcript levels at SNC1 and another defense response gene, RECOGNITION OF PERONOSPORA PARASITICA 4 (RPP4). Thus, interaction between MOS9 and ATXR7 induces defense-related gene expression via H3K4me3 deposition (Xia et al., 2013). Studies of two demethylases, Rice OsJMJ704 and Arabidopsis JMJ14, indicate that demethylation of H3K4me3 can play different roles in plant defense responses. Under Xanthomonas oryzae pv. Oryzae (Xoo) infection, transcription levels of $\mathrm{OsJMJ}^{7} 04$ were induced in rice plants andosjmj704 mutants were more sensitive to Xoo infection. The expression of negative defense regulator genes (NRR, OsWRKY62 and $\mathrm{Os}-11 \mathrm{~N} 3$, ,) and the ratio of $\mathrm{H} 3 \mathrm{~K} 4 \mathrm{me} 2 / \mathrm{H} 3 \mathrm{~K} 4 \mathrm{me} 3$ at these genes was increased in the osjmj704 mutant, suggesting a positive role for OsJMJ704 in rice bacterial defense by repressing negative defense-related genes through regulation of $\mathrm{H} 3 \mathrm{~K} 4 \mathrm{me} 2 / \mathrm{H} 3 \mathrm{~K} 4 \mathrm{me} 3$ levels (Hou et al., 2015). In Arabidopsis, jmj14 mutants were more sensitive to the Pst DC3000 bacterial pathogen. Under normal conditions, the transcript levels of positive defense-related genes (PR1, ALD1, FMO1, and WRKY 70 ) were not significantly changed in jmj14 mutants, but negative defense-related genes (e.g. SNI1 ) were up-regulated. H3K4me3 levels in jmj14 and wild-type plants at these genes positively correlated with the levels of gene expression; induced SNI1 injmj14 had higher levels of H3K4me3 at the TSS, and positive defense-related genes showed no changes in either transcripts or H3K4me3. After pathogen infection, the expression of positive defense-related genes were induced to a lesser degree in mutants compared to wild type, and H3K4me3 abundance at these genes was lower injmj14 mutants, suggesting JMJ14 is involved in H3K4me3 enrichment at specific genes during defense responses. Considering the H3K4 demethylase activity of JMJ14, a positive role of JMJ14 in enrichment of H3K4me3 at positive defense-related genes was unexpected. The identification of JMJ14 interacting partners and the genes directly targeted by JMJ14 during pathogen infection should help to resolve the roles of H3K4me3 demethylases during defense responses (D. Li et al., 2019).

Exogenous application of the phytohormone salicylic acid (SA), or certain synthetic SA analogs, is sufficient to induce resistance to a subsequent pathogen challenge, a phenomenon known as chemical priming (Kohler et al., 2002). The priming of defense genes poises them for a stronger transcriptional response to future pathogens relative to non-exposed plants. Interestingly, H3K4me3 levels increase at some defense genes during priming and persist after the removal of the initial stress signal (Jaskiewicz et al., 2011). This is similar to the transcriptional memory function described for trainable drought-responsive genes (Ding, Fromm, et al., 2012). Priming is negatively regulated by CHROMATIN ASSEMBLY FACTOR 1 (CAF1), which appears to antagonize H3K4me3 deposition at the TSS of defense genes (Mozgova et al., 2015a). Therefore, CAF1 may prevent ectopic defense gene activation.

\section{H3K4me3 Interactions with other Chromatin Modifications}

A central aspect of H3K4me3 function is its interaction with other factors, especially histone variants and other histone modifications. Among them, the H2A.Z histone variant and H3K27me3 have been well-studied and show distinct functional correlations with H3K4me3. H2A.Z is a conserved variant among eukaryotes that plays multiple roles in chromatin structure and gene expression (Jackson, Falciano, \& Gorovsky, 1996; Jarillo 
\& Pineiro, 2015). Genome-wide analyses showed that H2A.Z is preferentially associated with H3K4me3, while showing partial association with H3K27me3, in rice shoots, callus, and seedlings (Foroozani et al., 2019; K. Zhang et al., 2017). In addition, the combination of H2A.Z with either H3K4me3 or H3K27me3 correlated with differences in gene expression (K. Zhang et al., 2017). Co-localization of H2A.Z and H3K4me3 in genic regions has also been demonstrated in Arabidopsis (Dai et al., 2017; Sequeira-Mendes et al., 2014). On the other hand, activity of enhancers was repressed by H2A.Z through promoting H3K27me3 and antagonizing H3K4me3 deposition at enhancer regions (Dai et al., 2017). Interestingly, Arabidopsis H2A.Z appeared to facilitate H3K4 trimethylation at miR156 loci (Xu et al., 2018) but antagonize it at some anthocyanin biosynthetic genes (Cai et al., 2019), reflecting divergent and context-dependent interactions between H2A.Z and H3K4me3.

In plants, Polycomb Repressive Complex 2 (PRC2) is a methyltransferase responsible for the deposition of H3K27me3, which acts antagonistically to H3K4me3 (Engelhorn et al., 2017; X. Zhang et al., 2009). Although the distribution and interactions between these two modifications have been investigated, a conclusive pattern remains elusive. Genome-wide analysis of both marks within Arabidopsis seedlings seems to indicate a mutually exclusive relationship (X. Zhang et al., 2009). However, when considering specific dehydration stress-induced memory genes, H3K27me3 and H3K4me3 are found to be independent of one another, showing the capability of occurring at the same gene at the same time (N. Liu et al., 2014). Similarly, H3K4me3 and H3K27me3 have been found to co-localize at bivalent domains in cold stress-responsive genes in potato (Zeng et al., 2019), as well as in rice genes differentially-expressed by ionizing radiation (Pan et al., 2017) or salt stress (Zheng et al., 2019). In each case, the correlation existed for both up- and down-regulated genes, consistent with H3K4me3/H3K27me3 bivalent domains allowing for greater access of transcriptional regulators (i.e. activators or repressors) in response to stress. Our recent work in rice suggests that H3K4me3/H3K27me3 bivalent nucleosomes also interact with H2A.Z to modulate both up- and down-regulation of phosphate-starvation response genes (Foroozani et al., 2019). In addition to modulating responses to stress, H3K4me3-H3K27me3 bivalent domains also play a role in developmental processes in Arabidopsis, including gametophyte development (Berr et al., 2010), flowering time regulation (Qian et al., 2018), and seed germination (Molitor, $\mathrm{Bu}, \mathrm{Yu}, \&$ Shen, 2014), and possibly in xylem tissue development inEucalyptus grandis (Hussey, Mizrachi, Groover, Berger, \& Myburg, 2015).

In addition to associations with other histone modifications and histone variants, H3K4me3 function is influenced by DNA methylation. Three DNA methylation pathways exist in plants to methylate DNA in distinct sequence contexts: CG, CHH, and CHG (X. Zhang et al., 2009). In Arabidopsis, CHG and CHH methylation were depleted in H3K4 mono/di/trimethylated genomic regions, whereas CG methylation was enriched at H3K4me1 regions (X. Zhang et al., 2009). The mutually exclusive relationship between DNA methylation and $\mathrm{H} 3 \mathrm{~K} 4 \mathrm{me} 2 / 3$ was further demonstrated in studies of demethylase mutants. In jmj14 plants, genes targeted by the RNA-directed DNA methylation (RdDM) pathway, which is responsible for de novo DNA methylation and maintenance of $\mathrm{CHH}$ and CHG methylation, exhibited reduced DNA methylation and elevated H3K4me3 levels (Deleris et al., 2010). Also, target genes of LDL1 and LDL2 demethylases exhibited increased expression and decreased non-CG DNA methylation in ldl1 ldl2 double mutants (D. Jiang et al., 2007). Compared to the ldl1 ldl2 double mutant, ajmj14 ldl1 ldl2 triple mutant showed an even greater reduction in DNA methylation, which was accompanied by increased levels of H3K4me2/3 (Greenberg et al., 2013). Together these studies indicate that H3K4me3 influences gene expression, in part, by antagonizing CHG and CHH methylation.

\section{Concluding Remarks}

The distribution of H3K4me3 at genic regions, particularly at the 5' end of genes, is conserved among species and implicates this histone modification in the regulation of gene expression. Based on evidence from a number of studies, H3K4me3 plays key roles in development and stress responses in plants. Elucidating the extent to which H3K4me3 directly regulates a given biological pathway, and by what mechanisms, has been challenging, although some patterns have emerged. The formation of the ATX1-containing COMPASSlike complex at the promoter is important for transcription initiation, whereas H3K4me3 deposited at the 
promoter-proximal and gene body regions facilitates transcription elongation and mRNA maturation, which are influenced by the breadth of H3K4me3 distribution. Therefore, ATX1 and H3K4me3 likely play important roles in the fundamental aspects of transcription for numerous genes. The expression of many genes is sensitive to developmental and/or environmental cues, and H3K4me3 also plays a role in integrating these signals. In particular, H3K4me3 associated with H3K27me3 in bivalent domains modulates expression of many genes that regulate developmental transitions, such as flowering (e.g. $S O C 1$ and $F L C$ ), shoot maturation (e.g. MIR156A and MIR156C), and gametophyte development (e.g. SPL / NZZ and MS1). Notably, the SHL protein exhibits dual recognition of both H3K4me3 and H3K27me3, and likely functions during many developmental transitions. H3K4me3-H3K27me3 bivalent domains serve as an integrator of not only developmental cues but also environmental signals. Although possibly a central part of virtually all stress-response pathways, H3K4me3-H3K27me3 bivalent domains have been shown to influence stressresponsive genes related to cold stress, ionizing radiation, and salt stress. Finally H3K4me3 dynamics have been linked to transcriptional memory of stress-responsive genes related to drought and heat stress, as well as transcriptional priming of pathogen-responsive genes. As with H3K4me3-H3K27me3 bivalent domains, H3K4me3-mediated memory is likely to be fundamental to many environmental stressors. As sessile organisms with sophisticated signaling networks that guide developmental processes and stress adaptations, plants represent an ideal system for further investigation into H3K4me3-dependent transcriptional and memory phenomena. Future studies can be strengthened by a number of considerations, including the use of single cell types, quantification of many marks simultaneously, particularly H3K4me2, H3K4me3 and H3K27me3, and at multiple genic regions, as well as temporal examination at conditions before, during, and after reversal of the stimulus. The continued identification and characterization of binding partners of HKMTs and JMJs, as well as factors that confer target-gene specificity are also necessary. These efforts will shed light on key unanswered questions such as the extent to which H3K4me3-mediated mechanisms are causal or effectual, and whether these mechanisms exhibit differences among diverse environmental stressors, distinct cell types, and different species.

\section{References}

Alonso, C., Ramos-Cruz, D., \& Becker, C. (2019). The role of plant epigenetics in biotic interactions. New Phytologist, 221 (2), 731-737.

Alvarez-Venegas, R., Abdallat, A. A., Guo, M., Alfano, J. R., \& Avramova, Z. (2007). Epigenetic control of a transcription factor at the cross section of two antagonistic pathways. Epigenetics, 2 (2), 106-113.

Alvarez-Venegas, R., \& Avramova, Z. (2005). Methylation patterns of histone H3 Lys 4, Lys 9 and Lys 27 in transcriptionally active and inactive Arabidopsis genes and in atx1 mutants. Nucleic Acids Research, 33 (16), 5199-5207.

Alvarez-Venegas, R., Pien, S., Sadder, M., Witmer, X., Grossniklaus, U., \& Avramova, Z. (2003). ATX-1, an Arabidopsis homolog of trithorax, activates flower homeotic genes. Current Biology, 13 (8), 627-637.

Avramova, Z. (2009). Evolution and pleiotropy of TRITHORAX function in Arabidopsis.

Barski, A., Cuddapah, S., Cui, K., Roh, T.-Y., Schones, D. E., Wang, Z., . . . Zhao, K. (2007). Highresolution profiling of histone methylations in the human genome. Cell, 129 (4), 823-837.

Bellegarde, F., Maghiaoui, A., Boucherez, J., Krouk, G., Lejay, L., Bach, L., . . . Martin, A. (2019). The Chromatin Factor HNI9 and ELONGATED HYPOCOTYL5 Maintain ROS Homeostasis under High Nitrogen Provision. Plant Physiol, 180 (1), 582-592. doi:10.1104/pp.18.01473

Bernstein, B. E., Humphrey, E. L., Erlich, R. L., Schneider, R., Bouman, P., Liu, J. S., . . . Schreiber, S. L. (2002). Methylation of histone H3 Lys 4 in coding regions of active genes. Proceedings of the National Academy of Sciences, 99 (13), 8695-8700.

Bernstein, B. E., Mikkelsen, T. S., Xie, X., Kamal, M., Huebert, D. J., Cuff, J., . . . Lander, E. S. (2006). A bivalent chromatin structure marks key developmental genes in embryonic stem cells. Cell, 125 (2), 315-326. 
doi:10.1016/j.cell.2006.02.041

Berr, A., McCallum, E. J., Menard, R., Meyer, D., Fuchs, J., Dong, A., \& Shen, W.-H. (2010). Arabidopsis SET DOMAIN GROUP2 is required for H3K4 trimethylation and is crucial for both sporophyte and gametophyte development. The plant cell, 22 (10), 3232-3248.

Berr, A., Xu, L., Gao, J., Cognat, V., Steinmetz, A., Dong, A., \& Shen, W.-H. (2009). SET DOMAIN GROUP25 encodes a histone methyltransferase and is involved in FLOWERING LOCUS C activation and repression of flowering. Plant physiology, 151 (3), 1476-1485.

Brusslan, J. A., Alvarez-Canterbury, A. M. R., Nair, N. U., Rice, J. C., Hitchler, M. J., \& Pellegrini, M. (2012). Genome-wide evaluation of histone methylation changes associated with leaf senescence in Arabidopsis. PLoS One, 7 (3), e33151.

Brusslan, J. A., Bonora, G., Rus-Canterbury, A. M., Tariq, F., Jaroszewicz, A., \& Pellegrini, M. (2015). A genome-wide chronological study of gene expression and two histone modifications, H3K4me3 and H3K9ac, during developmental leaf senescence. Plant physiology, 168 (4), 1246-1261.

Cai, H., Zhang, M., Chai, M., He, Q., Huang, X., Zhao, L., \& Qin, Y. (2019). Epigenetic regulation of anthocyanin biosynthesis by an antagonistic interaction between H2A. Z and H3K4me3. New Phytologist, 221 (1), 295-308.

Calero, G., Wilson, K. F., Ly, T., Rios-Steiner, J. L., Clardy, J. C., \& Cerione, R. A. (2002). Structural basis of $\mathrm{m} 7 \mathrm{GpppG}$ binding to the nuclear cap-binding protein complex. Nature Structural 85 Molecular Biology, 9 (12), 912-917.

Carlone, D. L., \& Skalnik, D. G. (2001). CpG binding protein is crucial for early embryonic development. Molecular and cellular biology, 21 (22), 7601-7606.

Chandrika, N. N. P., Sundaravelpandian, K., Yu, S. M., \& Schmidt, W. (2013). ALFIN-LIKE 6 is involved in root hair elongation during phosphate deficiency in Arabidopsis. New Phytologist, 198 (3), 709-720.

Chen, K., Chen, Z., Wu, D., Zhang, L., Lin, X., Su, J., . . . Chen, X. (2015). Broad H3K4me3 is associated with increased transcription elongation and enhancer activity at tumor-suppressor genes. Nature genetics, $47(10), 1149$.

Chen, L.-T., Luo, M., Wang, Y.-Y., \& Wu, K. (2010). Involvement of Arabidopsi s histone deacetylase HDA6 in ABA and salt stress response. Journal of experimental botany, 61 (12), 3345-3353.

Chen, L., Luo, J., Cui, Z., Xue, M., Wang, L., Zhang, X., . . . He, Y. (2017). ATX3, ATX4, and ATX5 encode putative H3K4 methyltransferases and are critical for plant development. Plant physiology, pp. 01944.02016.

Chen, Q., Chen, X., Wang, Q., Zhang, F., Lou, Z., Zhang, Q., \& Zhou, D.-X. (2013). Structural basis of a histone $\mathrm{H} 3$ lysine 4 demethylase required for stem elongation in rice. PLoS genetics, 9 (1), e1003239.

Chen, X., Hu, Y., \& Zhou, D.-X. (2011). Epigenetic gene regulation by plant Jumonji group of histone demethylase. Biochimica et Biophysica Acta (BBA)-Gene Regulatory Mechanisms, 1809 (8), 421-426.

Choi, S. C., Lee, S., Kim, S.-R., Lee, Y.-S., Liu, C., Cao, X., \& An, G. (2014). Trithorax group protein Oryza sativa Trithorax1 controls flowering time in rice via interaction with early heading date3.Plant physiology, 164 (3), 1326-1337.

Clouaire, T., Webb, S., Skene, P., Illingworth, R., Kerr, A., Andrews, R., . . . Bird, A. (2012). Cfp1 integrates both $\mathrm{CpG}$ content and gene activity for accurate H3K4me3 deposition in embryonic stem cells. Genes $\&$ development, 26 (15), 1714-1728.

Crevillen, P., Yang, H., Cui, X., Greeff, C., Trick, M., Qiu, Q., . . . Dean, C. (2014). Epigenetic reprogramming that prevents transgenerational inheritance of the vernalized state. Nature, 515 (7528), 
587-590. doi:10.1038/nature13722

Cruz, C., Della Rosa, M., Krueger, C., Gao, Q., Horkai, D., King, M., . . . Houseley, J. (2018). Trimethylation of histone H3 lysine 4 facilitates gene expression in ageing cells. Elife, 7, e34081.

Cui, P., Liu, W., Zhao, Y., Lin, Q., Ding, F., Xin, C., . . . Yu, J. (2012). The association between H3K4me3 and antisense transcription. Genomics Proteomics Bioinformatics, 10 (2), 74-81. doi:10.1016/j.gpb.2012.05.001

D'Urso, A., Takahashi, Y. H., Xiong, B., Marone, J., Coukos, R., Randise-Hinchliff, C., . . . Brickner, J. H. (2016). Set1/COMPASS and Mediator are repurposed to promote epigenetic transcriptional memory.elife, 5 . doi:10.7554/eLife.16691

Dai, X., Bai, Y., Zhao, L., Dou, X., Liu, Y., Wang, L., . . . Huang, X. (2017). H2A. Z Represses Gene Expression by Modulating Promoter Nucleosome Structure and Enhancer Histone Modifications in Arabidopsis.Molecular plant, 10 (10), 1274-1292.

Deleris, A., Greenberg, M. V., Ausin, I., Law, R. W., Moissiard, G., Schubert, D., \& Jacobsen, S. E. (2010). Involvement of a Jumonji-C domain-containing histone demethylase in DRM2-mediated maintenance of DNA methylation. EMBO reports, 11 (12), 950-955.

Ding, Y., Avramova, Z., \& Fromm, M. (2011a). The Arabidopsis trithorax-like factor ATX1 functions in dehydration stress responses via ABA-dependent and ABA-independent pathways. The Plant Journal, 66 (5), 735-744.

Ding, Y., Avramova, Z., \& Fromm, M. (2011b). Two distinct roles of ARABIDOPSIS HOMOLOG OF TRITHORAX1 (ATX1) at promoters and within transcribed regions of ATX1-regulated genes. The plant cell, 23 (1), 350-363.

Ding, Y., Fromm, M., \& Avramova, Z. (2012). Multiple exposures to drought'train'transcriptional responses in Arabidopsis. Nature Communications, 3 , 740.

Ding, Y., Lapko, H., Ndamukong, I., Xia, Y., Al-Abdallat, A., Lalithambika, S., . . . Riethoven, J.-J. (2009). TheArabidopsis Chromatin Modifier ATX1, the Myotubularin-like AtMTM, and the response to Drought; a view from the other end of the pathway. Plant signaling \& behavior, 4 (11), 1049-1058.

Ding, Y., Ndamukong, I., Xu, Z., Lapko, H., Fromm, M., \& Avramova, Z. (2012a). ATX1-generated H3K4me3 is required for efficient elongation of transcription, not initiation, at ATX1-regulated genes. PLoS Genet, 8 (12), e1003111.

Ding, Y., Ndamukong, I., Xu, Z., Lapko, H., Fromm, M., \& Avramova, Z. (2012b). ATX1-generated H3K4me3 is required for efficient elongation of transcription, not initiation, at ATX1-regulated genes. PLoS genetics, 8 (12), e1003111.

Engelhorn, J., Blanvillain, R., Kroner, C., Parrinello, H., Rohmer, M., Pose, D., . . . Carles, C. C. (2017). Dynamics of H3K4me3 chromatin marks prevails over H3K27me3 for gene regulation during flower morphogenesis in Arabidopsis thaliana. Epigenomes, 1 (2), 8.

Foroozani, M., Zahraeifard, S., Oh, D.-H., Wang, G., Dassanayake, M., \& Smith, A. (2019). Defining chromatin state transitions predicts a network that modulates cell wall remodeling in phosphate-starved rice shoots. bioRxiv , 706507. doi:10.1101/706507

Greenberg, M. V., Deleris, A., Hale, C. J., Liu, A., Feng, S., \& Jacobsen, S. E. (2013). Interplay between active chromatin marks and RNA-directed DNA methylation in Arabidopsis thaliana. PLoS genetics, 9 (11), e1003946.

Greer, E. L., \& Shi, Y. (2012). Histone methylation: a dynamic mark in health, disease and inheritance. Nat Rev Genet, 13 (5), 343-357. doi:10.1038/nrg3173 
Guo, L., Yu, Y., Law, J. A., \& Zhang, X. (2010a). SET DOMAIN GROUP2 is the major histone H3 lysie 4 trimethyltransferase in Arabidopsis.Proceedings of the National Academy of Sciences , 201010478.

Guo, L., Yu, Y., Law, J. A., \& Zhang, X. (2010b). SET DOMAIN GROUP2 is the major histone H3 lysine 4 trimethyltransferase in Arabidopsis.Proceedings of the National Academy of Sciences, 107 (43), 18557-18562.

Hou, Y., Wang, L., Wang, L., Liu, L., Li, L., Sun, L., . . . Huang, S. (2015). JMJ704 positively regulates rice defense response against Xanthomonas oryzae pv. oryzae infection via reducing H3K4me2/3 associated with negative disease resistance regulators. BMC plant biology, 15 (1), 286.

Howe, F. S., Boubriak, I., Sale, M. J., Nair, A., Clynes, D., Grijzenhout, A., . . . Mellor, J. (2014). Lysine acetylation controls local protein conformation by influencing proline isomerization. Mol Cell, 55 (5), 733-744. doi:10.1016/j.molcel.2014.07.004

Howe, F. S., Fischl, H., Murray, S. C., \& Mellor, J. (2017). Is H3K4me3 instructive for transcription activation? Bioessays, 39 (1), 1-12.

Huang, S., Zhang, A., Jin, J. B., Zhao, B., Wang, T. J., Wu, Y., . . . Guo, P. (2019). Arabidopsis histone H3K4 demethylase JMJ 17 functions in dehydration stress response. New Phytologist, 223 (3), 1372-1387.

Hussey, S. G., Mizrachi, E., Groover, A., Berger, D. K., \& Myburg, A. A. (2015). Genome-wide mapping of histone H3 lysine 4 trimethylation in Eucalyptus grandis developing xylem. BMC plant biology, 15 (1), 117.

Jackson, J. D., Falciano, V. T., \& Gorovsky, M. A. (1996). A likely histone H2A. F/Z variant in Saccharomyces cerevisiae. Trends in biochemical sciences, 21 (12), 466-467.

Jarillo, J. A., \& Pineiro, M. (2015). H2A.Z mediates different aspects of chromatin function and modulates flowering responses in Arabidopsis.Plant J, 83 (1), 96-109. doi:10.1111/tpj.12873

Jaskiewicz, M., Conrath, U., \& Peterhansel, C. (2011). Chromatin modification acts as a memory for systemic acquired resistance in the plant stress response. EMBO reports, 12 (1), 50-55.

Jeong, J.-H., Song, H.-R., Ko, J.-H., Jeong, Y.-M., Kwon, Y. E., Seol, J. H., . . . Noh, Y.-S. (2009). Repression of FLOWERING LOCUS T chromatin by functionally redundant histone H3 lysine 4 demethylases in Arabidopsis. PLoS One, 4 (11), e8033.

Jiang, D., Kong, N. C., Gu, X., Li, Z., \& He, Y. (2011). Arabidopsis COMPASS-like complexes mediate histone H3 lysine-4 trimethylation to control floral transition and plant development. PLoS Genet, 7 (3), e1001330.

Jiang, D., Yang, W., He, Y., \& Amasino, R. M. (2007). Arabidopsis relatives of the human lysine-specific Demethylase1 repress the expression of FWA and FLOWERING LOCUS C and thus promote the floral transition. The plant cell, 19 (10), 2975-2987.

Jiang, P., Wang, S., Zheng, H., Li, H., Zhang, F., Su, Y., . . . Ding, Y. (2018). SIP 1 participates in regulation of flowering time in rice by recruiting OsTrx1 to Ehd1. New Phytologist, 219 (1), 422-435.

Kim, J.-M., To, T. K., Ishida, J., Morosawa, T., Kawashima, M., Matsui, A., . . . Seki, M. (2008). Alterations of lysine modifications on the histone $\mathrm{H} 3 \mathrm{~N}$-tail under drought stress conditions in Arabidopsis thaliana. Plant and Cell Physiology, 49 (10), 1580-1588.

Kohler, A., Schwindling, S., \& Conrath, U. (2002). Benzothiadiazole-induced priming for potentiated responses to pathogen infection, wounding, and infiltration of water into leaves requires the NPR1/NIM1 gene in Arabidopsis. Plant physiology, 128 (3), 1046-1056.

Lamke, J., Brzezinka, K., Altmann, S., \& Baurle, I. (2016). A hit-and-run heat shock factor governs sustained histone methylation and transcriptional stress memory. The EMBO journal, 35 (2), 162-175.

Lee, B. B., Choi, A., Kim, J. H., Jun, Y., Woo, H., Ha, S. D., . . . Buratowski, S. (2018). Rpd3L HDAC links H3K4me3 to transcriptional repression memory. Nucleic acids research, 46 (16), 8261-8274. 
Lee, I., Michaels, S. D., Masshardt, A. S., \& Amasino, R. M. (1994). The late-flowering phenotype of FRIGIDA and mutations in LUMINIDEPENDENS is suppressed in the Landsberg erecta strain of Arabidopsis. The Plant Journal, 6 (6), 903-909.

Lee, K., Park, O.-S., Choi, C. Y., \& Seo, P. J. (2019). ARABIDOPSIS TRITHORAX 4 Facilitates Shoot Identity Establishment during the Plant Regeneration Process. Plant and Cell Physiology, 60 (4), 826-834.

Lee, W. Y., Lee, D., Chung, W. I., \& Kwon, C. S. (2009). Arabidopsis ING and Alfin1-like protein families localize to the nucleus and bind to H3K4me3/2 via plant homeodomain fingers. The Plant Journal, 58 (3), 511-524.

Lenstra, T. L., Benschop, J. J., Kim, T., Schulze, J. M., Brabers, N. A., Margaritis, T., . . Koerkamp, M. J. G. (2011). The specificity and topology of chromatin interaction pathways in yeast. Molecular cell, 42 (4), 536-549.

Li, D., Liu, R., Singh, D., Yuan, X., Kachroo, P., \& Raina, R. (2019). JMJ14 encoded H3K4 demethylase modulates immune responses by regulating defence gene expression and pipecolic acid levels. New Phytologist

Li, X., Wang, X., He, K., Ma, Y., Su, N., He, H., . . . Jiang, J. (2008). High-resolution mapping of epigenetic modifications of the rice genome uncovers interplay between DNA methylation, histone methylation, and gene expression. The plant cell, 20 (2), 259-276.

Li, Y., Mukherjee, I., Thum, K. E., Tanurdzic, M., Katari, M. S., Obertello, M., . . . Coruzzi, G. M. (2015). The histone methyltransferase SDG8 mediates the epigenetic modification of light and carbon responsive genes in plants. Genome Biol, 16 , 79. doi:10.1186/s13059-015-0640-2

Li, Z., Jiang, D., Fu, X., Luo, X., Liu, R., \& He, Y. (2016). Coupling of histone methylation and RNA processing by the nuclear mRNA cap-binding complex. Nature plants, 2 , 16015.

Liu, C., Lu, F., Cui, X., \& Cao, X. (2010). Histone methylation in higher plants. Annual review of plant biology, $61,395-420$.

Liu, C. L., Kaplan, T., Kim, M., Buratowski, S., Schreiber, S. L., Friedman, N., \& Rando, O. J. (2005). Single-nucleosome mapping of histone modifications in S. cerevisiae. PLoS biology, 3 (10), e328.

Liu, K., Yu, Y., Dong, A., \& Shen, W. H. (2017). SET DOMAIN GROUP701 encodes a H3K4-methytransferase and regulates multiple key processes of rice plant development. New Phytologist .

Liu, N., Fromm, M., \& Avramova, Z. (2014). H3K27me3 and H3K4me3 chromatin environment at superinduced dehydration stress memory genes of Arabidopsis thaliana. Molecular plant, 7 (3), 502-513.

Liu, P., Zhang, S., Zhou, B., Luo, X., Zhou, X. F., Cai, B., . . . Cao, X. (2019). The histone H3K4 demethylase JMJ16 represses leaf senescence in Arabidopsis. The plant cell, 31 (2), 430-443.

Liu, X., Zhou, S., Wang, W., Ye, Y., Zhao, Y., Xu, Q., . . . Zhou, D.-X. (2015). Regulation of histone methylation and reprogramming of gene expression in the rice inflorescence meristem. The plant cell, 27 (5), 1428-1444.

Lopez-Gonzalez, L., Mouriz, A., Narro-Diego, L., Bustos, R., Martinez-Zapater, J. M., Jarillo, J. A., \& Pineiro, M. (2014). Chromatin-dependent repression of the Arabidopsis floral integrator genes involves plant specific PHD-containing proteins. Plant Cell, 26 (10), 3922-3938. doi:10.1105/tpc.114.130781

Lu, F., Cui, X., Zhang, S., Jenuwein, T., \& Cao, X. (2011). Arabidopsis REF6 is a histone H3 lysine 27 demethylase. Nat Genet, 43 (7), 715-719. doi:10.1038/ng.854

Lu, F., Cui, X., Zhang, S., Liu, C., \& Cao, X. (2010). JMJ14 is an H3K4 demethylase regulating flowering time in Arabidopsis. Cell research, 20 (3), 387. 
Luger, K., Mader, A. W., Richmond, R. K., Sargent, D. F., \& Richmond, T. J. (1997). Crystal structure of the nucleosome core particle at 2.8 A resolution. Nature, 389 (6648), 251.

Malapeira, J., Khaitova, L. C., \& Mas, P. (2012). Ordered changes in histone modifications at the core of the Arabidopsis circadian clock.Proceedings of the National Academy of Sciences, 109 (52), 21540-21545.

Michaels, S. D., \& Amasino, R. M. (1999). FLOWERING LOCUS C encodes a novel MADS domain protein that acts as a repressor of flowering. The Plant Cell, 11 (5), 949-956.

Mittler, R., Finka, A., \& Goloubinoff, P. (2012). How do plants feel the heat? Trends Biochem Sci, 37 (3), 118-125. doi:10.1016/j.tibs.2011.11.007

Molitor, A. M., Bu, Z., Yu, Y., \& Shen, W.-H. (2014). Arabidopsis AL PHD-PRC1 complexes promote seed germination through H3K4me3-to-H3K27me3 chromatin state switch in repression of seed developmental genes.PLoS genetics, 10 (1), e1004091.

Mozgova, I., Wildhaber, T., Liu, Q., Abou-Mansour, E., L'Haridon, F., Metraux, J.-P., . . . Hennig, L. (2015a). Chromatin assembly factor CAF-1 represses priming of plant defence response genes. Nature plants, $1(9), 1-8$.

Mozgova, I., Wildhaber, T., Liu, Q., Abou-Mansour, E., L'Haridon, F., Metraux, J.-P., . . . Hennig, L. (2015b). Chromatin assembly factor CAF-1 represses priming of plant defence response genes. Nature plants, 1 (9), 15127.

Murray, S. C., Lorenz, P., Howe, F., Wouters, M., Brown, T., Xi, S., . . . Angel, A. (2019). H3K4me3 is neither instructive for, nor informed by, transcription. bioRxiv , 709014.

Mussig, C., Kauschmann, A., Clouse, S. D., \& Altmann, T. (2000). The Arabidopsis PHD-finger protein SHL is required for proper development and fertility. Mol Gen Genet, 264 (4), 363-370. doi:10.1007/s004380000313

Napsucialy-Mendivil, S., Alvarez-Venegas, R., Shishkova, S., \& Dubrovsky, J. G. (2014). ARABIDOPSIS HOMOLOG of TRITHORAX1 (ATX1) is required for cell production, patterning, and morphogenesis in root development. Journal of experimental botany, 65 (22), 6373-6384.

Ng, D. W. K., Wang, T., Chandrasekharan, M. B., Aramayo, R., Kertbundit, S., \& Hall, T. C. (2007). Plant SET domain-containing proteins: Structure, function and regulation. Biochimica et Biophysica Acta (BBA) - Gene Structure and Expression, 1769 (5), 316-329. doi:http://dx.doi.org/10.1016/j.bbaexp.2007.04.003

Ning, Y.-Q., Ma, Z.-Y., Huang, H.-W., Mo, H., Zhao, T.-t., Li, L., . . . He, X.-J. (2015). Two novel NAC transcription factors regulate gene expression and flowering time by associating with the histone demethylase JMJ14. Nucleic Acids Research, 43 (3), 1469-1484.

Pan, X., Fang, Y., Yang, X., Zheng, D., Chen, L., Wang, L., . . . Cheng, Z. (2017). Chromatin states responsible for the regulation of differentially expressed genes under $60 \mathrm{Co}^{\sim} \gamma$ ray radiation in rice. $B M C$ genomics, 18 (1), 778.

Park, S., Kim, G. W., Kwon, S. H., \& Lee, J. S. (2020). Broad domains of histone H3 lysine 4 trimethylation in transcriptional regulation and disease. FEBS J . doi:10.1111/febs.15219

Pien, S., Fleury, D., Mylne, J. S., Crevillen, P., Inzé, D., Avramova, Z., . . . Grossniklaus, U. (2008). ARABIDOPSIS TRITHORAX1 dynamically regulates FLOWERING LOCUS C activation via histone 3 lysine 4 trimethylation. The plant cell, 20 (3), 580-588.

Pinon, V., Yao, X., Dong, A., \& Shen, W.-H. (2017). H3K4me3 is crucial for chromatin condensation and mitotic division during male gametogenesis. Plant physiology, pp. 00306.02017.

Poethig, R. S. (2010). The past, present, and future of vegetative phase change. Plant physiology, 154 (2), 541-544. 
Qian, S., Lv, X., Scheid, R. N., Lu, L., Yang, Z., Chen, W., . . Du, J. (2018). Dual recognition of H3K4me3 and H3K27me3 by a plant histone reader SHL. Nat Commun, 9 (1), 2425. doi:10.1038/s41467-018-04836-y

Ram, O., Goren, A., Amit, I., Shoresh, N., Yosef, N., Ernst, J., . . . Coyne, M. (2011). Combinatorial patterning of chromatin regulators uncovered by genome-wide location analysis in human cells. Cell, 147 (7), 1628-1639.

Roberts, M. R., \& Sánchez, A. L. (2019). Plant Epigenetic Mechanisms in Response to Biotic Stress. In Epigenetics in Plants of Agronomic Importance: Fundamentals and Applications (pp. 65-113): Springer.

Roguev, A., Schaft, D., Shevchenko, A., Pijnappel, W. P., Wilm, M., Aasland, R., \& Stewart, A. F. (2001). The Saccharomyces cerevisiae Set1 complex includes an Ash2 homologue and methylates histone 3 lysine 4. The EMBO journal, 20 (24), 7137-7148.

Saleh, A., Alvarez-Venegas, R., Yilmaz, M., Le, O., Hou, G., Sadder, M., . . . Ladunga, I. (2008). The highly similar Arabidopsis homologs of trithorax ATX1 and ATX2 encode proteins with divergent biochemical functions. The plant cell, 20 (3), 568-579.

Santos-Rosa, H., Schneider, R., Bannister, A. J., Sherriff, J., Bernstein, B. E., Emre, N. T., . . Kouzarides, T. (2002). Active genes are tri-methylated at K4 of histone H3. Nature, 419 (6905), 407-411.

Schneider, J., Wood, A., Lee, J.-S., Schuster, R., Dueker, J., Maguire, C., . . Shilatifard, A. (2005). Molecular regulation of histone $\mathrm{H} 3$ trimethylation by COMPASS and the regulation of gene expression. Molecular cell, 19 (6), 849-856.

Searle, I. R., Pontes, O., Melnyk, C. W., Smith, L. M., \& Baulcombe, D. C. (2010). JMJ14, a JmjC domain protein, is required for RNA silencing and cell-to-cell movement of an RNA silencing signal in Arabidopsis. Genes 83 development, 24 (10), 986-991.

Sequeira-Mendes, J., Aragüez, I., Peiró, R., Mendez-Giraldez, R., Zhang, X., Jacobsen, S. E., . . Gutierrez, C. (2014). The functional topography of the Arabidopsis genome is organized in a reduced number of linear motifs of chromatin states. The plant cell, 26 (6), 2351-2366.

Shen, Y., Conde e Silva, N., Audonnet, L., Servet, C., Wei, W., \& Zhou, D.-X. (2014). Over-expression of histone H3K4 demethylase gene JMJ15 enhances salt tolerance in Arabidopsis. Frontiers in plant science, 5 , 290 .

Shi, Y., Lan, F., Matson, C., Mulligan, P., Whetstine, J. R., Cole, P. A., . . . Shi, Y. (2004). Histone demethylation mediated by the nuclear amine oxidase homolog LSD1. Cell, 119 (7), 941-953.

Shilatifard, A. (2012). The COMPASS family of histone H3K4 methylases: mechanisms of regulation in development and disease pathogenesis. Annual review of biochemistry, 81 , 65-95.

Sokol, A., Kwiatkowska, A., Jerzmanowski, A., \& Prymakowska-Bosak, M. (2007). Up-regulation of stressinducible genes in tobacco and Arabidopsis cells in response to abiotic stresses and ABA treatment correlates with dynamic changes in histone H3 and H4 modifications. Planta, 227 (1), 245-254. doi:10.1007/s00425-0070612-1

Song, T., Zhang, Q., Wang, H., Han, J., Xu, Z., Yan, S., \& Zhu, Z. (2018). OsJMJ703, a rice histone demethylase gene, plays key roles in plant development and responds to drought stress. Plant Physiology and Biochemistry, 132, 183-188.

Song, Y., Ji, D., Li, S., Wang, P., Li, Q., \& Xiang, F. (2012). The dynamic changes of DNA methylation and histone modifications of salt responsive transcription factor genes in soybean. PLoS One, 7 (7), e41274.

Stief, A., Altmann, S., Hoffmann, K., Pant, B. D., Scheible, W. R., \& Baurle, I. (2014). Arabidopsis miR156 Regulates Tolerance to Recurring Environmental Stress through SPL Transcription Factors. Plant Cell, 26 (4), 1792-1807. doi:10.1105/tpc.114.123851 
Tamada, Y., Yun, J.-Y., chul Woo, S., \& Amasino, R. M. (2009). ARABIDOPSIS TRITHORAX-RELATED7 is required for methylation of lysine 4 of histone $\mathrm{H} 3$ and for transcriptional activation of FLOWERING LOCUS C. The plant cell, 21 (10), 3257-3269.

Tsuji, H., Saika, H., Tsutsumi, N., Hirai, A., \& Nakazono, M. (2006). Dynamic and reversible changes in histone H3-Lys4 methylation and $\mathrm{H} 3$ acetylation occurring at submergence-inducible genes in rice.Plant and Cell Physiology, 47 (7), 995-1003.

Tsukada, Y.-i., Fang, J., Erdjument-Bromage, H., Warren, M. E., Borchers, C. H., Tempst, P., \& Zhang, Y. (2006). Histone demethylation by a family of JmjC domain-containing proteins. Nature, 439 (7078), 811.

Ueda, M., \& Seki, M. (2020). Histone modifications form epigenetic regulatory networks to regulate abiotic stress response. Plant physiology, 182 (1), 15.

van Dijk, K., Ding, Y., Malkaram, S., Riethoven, J.-J. M., Liu, R., Yang, J., . . . Ladunga, I. (2010). Dynamic changes in genome-wide histone $\mathrm{H} 3$ lysine 4 methylation patterns in response to dehydration stress in Arabidopsis thaliana. BMC plant biology, 10 (1), 238.

Vastenhouw, N. L., \& Schier, A. F. (2012). Bivalent histone modifications in early embryogenesis. Curr Opin Cell Biol, 24 (3), 374-386. doi:10.1016/j.ceb.2012.03.009

Widiez, T., Girin, T., Berr, A., Ruffel, S., Krouk, G., Vayssières, A., . . Lepetit, M. (2011). HIGH NITROGEN INSENSITIVE 9 (HNI9)-mediated systemic repression of root NO3- uptake is associated with changes in histone methylation. Proceedings of the National Academy of Sciences, 108 (32), 13329-13334.

Xia, S., Cheng, Y. T., Huang, S., Win, J., Soards, A., Jinn, T.-L., . . . Zhang, Y. (2013). Regulation of transcription of nucleotide-binding leucine-rich repeat-encoding genes SNC1 and RPP4 via H3K4 trimethylation. Plant physiology, 162 (3), 1694-1705.

Xu, M., Leichty, A. R., Hu, T., \& Poethig, R. S. (2018). H2A. Z promotes the transcription of MIR156A and MIR156C in Arabidopsis by facilitating the deposition of H3K4me3. Development, 145 (2), dev152868.

Yang, H., Han, Z., Cao, Y., Fan, D., Li, H., Mo, H., . . . Yue, Y. (2012). A companion cell-dominant and developmentally regulated H3K4 demethylase controls flowering time in Arabidopsis via the repression of FLC expression. PLoS genetics, 8 (4), e1002664.

Yang, H., Mo, H., Fan, D., Cao, Y., Cui, S., \& Ma, L. (2012). Overexpression of a histone H3K4 demethylase, JMJ15, accelerates flowering time in Arabidopsis. Plant cell reports, 31 (7), 1297-1308.

Yang, W., Jiang, D., Jiang, J., \& He, Y. (2010). A plant-specific histone H3 lysine 4 demethylase represses the floral transition in Arabidopsis. The Plant Journal, 62 (4), 663-673.

Yao, X., Feng, H., Yu, Y., Dong, A., \& Shen, W.-H. (2013). SDG2-mediated H3K4 methylation is required for proper Arabidopsis root growth and development. PLoS One, 8 (2), e56537.

Yokoo, T., Saito, H., Yoshitake, Y., Xu, Q., Asami, T., Tsukiyama, T., . . . Tanisaka, T. (2014). Se14, encoding a JmjC domain-containing protein, plays key roles in long-day suppression of rice flowering through the demethylation of H3K4me3 of RFT1. PLoS One, 9 (4), e96064.

You, Y., Sawikowska, A., Neumann, M., Pose, D., Capovilla, G., Langenecker, T., . . . Schmid, M. (2017). Temporal dynamics of gene expression and histone marks at the Arabidopsis shoot meristem during flowering. Nature Communications, 8, 15120.

Yu, S., Cao, L., Zhou, C.-M., Zhang, T.-Q., Lian, H., Sun, Y., . . . Wang, J.-W. (2013). Sugar is an endogenous cue for juvenile-to-adult phase transition in plants. elife, 2 , e00269.

Yun, J.-Y., Tamada, Y., Kang, Y. E., \& Amasino, R. M. (2012). Arabidopsis trithorax-related3/SET domain GROUP2 is required for the winter-annual habit of Arabidopsis thaliana. Plant and Cell Physiology, 53 (5), 834-846. 
Zeng, Z., Zhang, W., Marand, A. P., Zhu, B., Buell, C. R., \& Jiang, J. (2019). Cold stress induces enhanced chromatin accessibility and bivalent histone modifications H3K4me3 and H3K27me3 of active genes in potato. Genome biology, 20 (1), 123.

Zhang, K., Xu, W., Wang, C., Yi, X., Zhang, W., \& Su, Z. (2017). Differential deposition of H2A. Z in combination with histone modifications within related genes in Oryza sativa callus and seedling. The Plant Journal, 89 (2), 264-277.

Zhang, X., Bernatavichute, Y. V., Cokus, S., Pellegrini, M., \& Jacobsen, S. E. (2009). Genome-wide analysis of mono-, di-and trimethylation of histone H3 lysine 4 in Arabidopsis thaliana.Genome biology, 10 (6), R62.

Zhang, Z., Shi, L., Dawany, N., Kelsen, J., Petri, M. A., \& Sullivan, K. E. (2016). H3K4 tri-methylation breadth at transcription start sites impacts the transcriptome of systemic lupus erythematosus. Clin Epigenetics, 8 , 14. doi:10.1186/s13148-016-0179-4

Zheng, D., Wang, L., Chen, L., Pan, X., Lin, K., Fang, Y., . . . Zhang, W. (2019). Salt-Responsive Genes are Differentially Regulated at the Chromatin Levels Between Seedlings and Roots in Rice. Plant and Cell Physiology .

Zhou, B. O., \& Zhou, J.-Q. (2011). Recent transcription-induced histone H3 lysine 4 (H3K4) methylation inhibits gene reactivation. Journal of biological chemistry, 286 (40), 34770-34776.

Zhou, H., Liu, Y., Liang, Y., Zhou, D., Li, S., Lin, S., . . . Huang, L. (2020). The function of the histone lysine methylation related SET domain group (SDG) proteins in plants. Protein Science .

Zong, W., Zhong, X., You, J., \& Xiong, L. (2013). Genome-wide profiling of histone H3K4-tri-methylation and gene expression in rice under drought stress. Plant molecular biology, 81 (1-2), 175-188. 

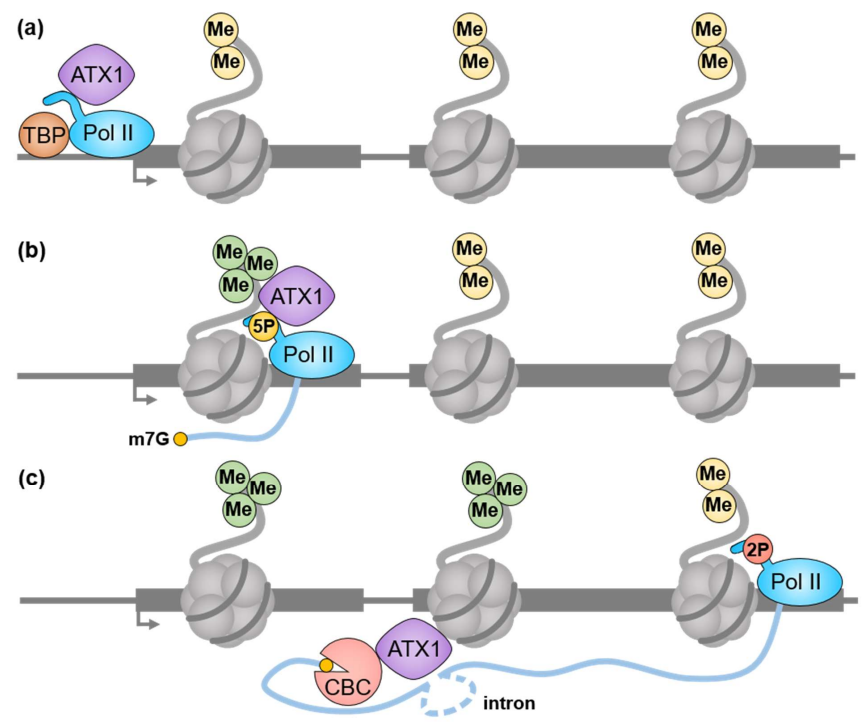

Figure 1. Functions of $\mathrm{H} 3 \mathrm{~K} 4 \mathrm{me} 3$ in transcriptional processes in plants. (a) The formation of the COMPASS-like complex, which includes the ATX1 H3K4 methyltransferase, facilitates assembly of the pre-initiation complex, including TATA Binding Protein (TBP) and RNA Polymerase II (Pol II), thereby regulating transcription initiation. (b) ATX1 is also recruited downstream of the transcription start site (TSS; indicated by the gray arrow) by the Ser5P (5P) early elongation form of Pol II, where it catalyzes H3K4 trimethylation. (c) Continued elongation results in a transition of Pol II to the Ser2P (2P) form, which releases ATX1 due to a lack of affinity to this form. ATX1 binds the mRNA cap-binding complex (CBC) to facilitate both preservation of the 5' 7-methylguanylate cap and mRNA splicing of introns. 


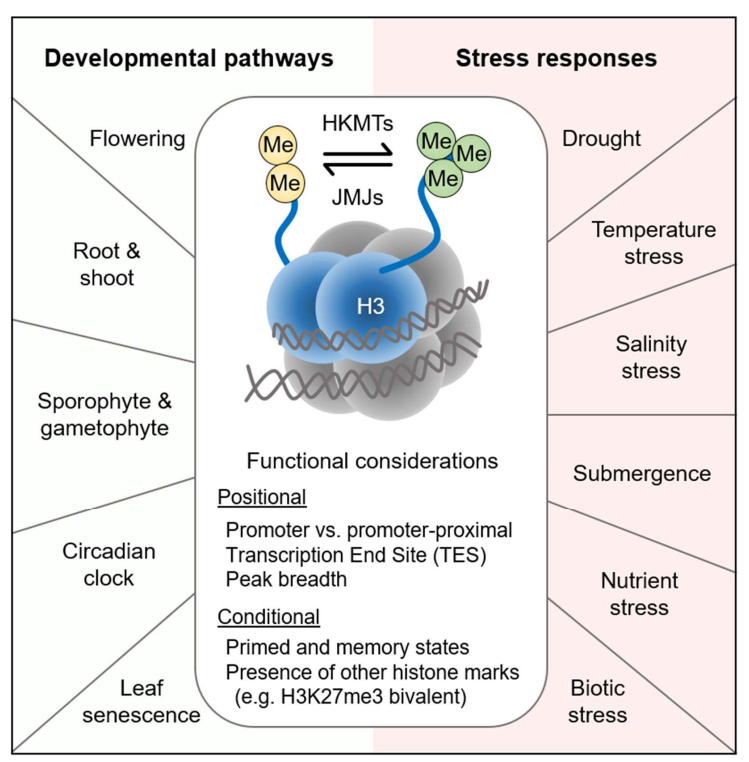

Figure 2. H3K4me3 plays important roles in diverse developmental pathways and stress responses. A number of histone lysine methyltransferases (HKMTs) and jumonji C-domain proteins (JMJs) catalyze methylation and demethylation of $\mathrm{H} 3 \mathrm{~K} 4$, respectively. The particular role of $\mathrm{H} 3 \mathrm{~K} 4 \mathrm{me} 3$ in modulating the developmental pathways and stress responses shown is dependent on several functional considerations that are either positional or conditional. 